Interim Report

IR-07-048

\title{
A New Mechanism for Recurrent Adaptive Radiations
}

Hiroshi Ito (itoh9@dolphin.c.u-tokyo.ac.jp)

Ulf Dieckmann (dieckmann@iiasa.ac.at)

\section{Approved by}

Leen Hordijk

Director, IIASA

December 2007 Institute, its National Member Organizations, or other organizations supporting the work. 


\section{IIASA STUDIES IN ADAPTIVE DYNAMICS No. 134}

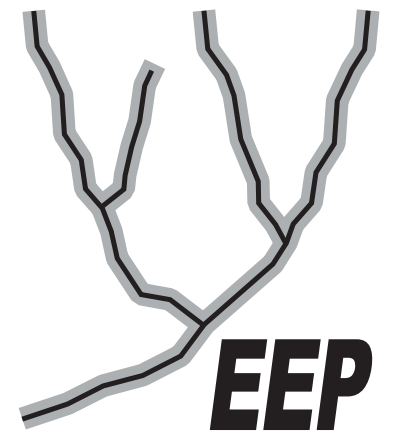

The Evolution and Ecology Program at IIASA fosters the development of new mathematical and conceptual techniques for understanding the evolution of complex adaptive systems.

Focusing on these long-term implications of adaptive processes in systems of limited growth, the Evolution and Ecology Program brings together scientists and institutions from around the world with IIASA acting as the central node.

Scientific progress within the network is collected in the IIASA Studies in Adaptive Dynamics series.
No. 1 Metz JAJ, Geritz SAH, Meszéna G, Jacobs FJA, van Heerwaarden JS: Adaptive Dynamics: A Geometrical Study of the Consequences of Nearly Faithful Reproduction. IIASA Working Paper WP-95-099 (1995). van Strien SJ, Verduyn Lunel SM (eds): Stochastic and Spatial Structures of Dynamical Systems, Proceedings of the Royal Dutch Academy of Science (KNAW Verhandelingen), North Holland, Amsterdam, pp. 183-231 (1996).

No. 2 Dieckmann U, Law R: The Dynamical Theory of Coevolution: A Derivation from Stochastic Ecological Processes. IIASA Working Paper WP-96-001 (1996). Journal of Mathematical Biology 34:579-612 (1996).

No. 3 Dieckmann U, Marrow P, Law R: Evolutionary Cycling of Predator-PreyInteractions: Population Dynamics and the Red Queen. IIASA Preprint (1995). Journal of Theoretical Biology 176:91-102 (1995).

No. 4 Marrow P, Dieckmann U, Law R: Evolutionary Dynamics of Predator-Prey Systems: An Ecological Perspective. IIASA Working Paper WP-96-002 (1996). Journal of Mathematical Biology 34:556-578 (1996).

No. 5 Law R, Marrow P, Dieckmann U: On Evolution under Asymmetric Competition. IIASA Working Paper WP-96-003 (1996). Evolutionary Ecology 11:485-501 (1997).

No. 6 Metz JAJ, Mylius SD, Diekmann O: When Does Evolution Optimize? On the Relation Between Types of Density Dependence and Evolutionarily Stable Life History Parameters. IIASA Working Paper WP-96-004 (1996).

No. 7 Ferrière R, Gatto M: Lyapunov Exponents and the Mathematics of Invasion in Oscillatory or Chaotic Populations. Theoretical Population Biology 48:126-171 (1995).

No. 8 Ferrière R, Fox GA: Chaos and Evolution. IIASA Preprint (1996). Trends in Ecology and Evolution 10:480485 (1995).

No. 9 Ferrière R, Michod RE: The Evolution of Cooperation in Spatially Heterogeneous Populations. IIASA Working Paper WP-96-029 (1996). The American Naturalist 147:692717 (1996).

No. 10 van Dooren TJM, Metz JAJ: Delayed Maturation in Temporally Structured Populations with Non-Equilibrium Dynamics. IIASA Working Paper WP-96-070 (1996). Journal of Evolutionary Biology 11:41-62 (1998).
No. 11 Geritz SAH, Metz JAJ, Kisdi É, Meszéna G: The Dynamics of Adaptation and Evolutionary Branching. IIASA Working Paper WP-96-077 (1996). Physical Review Letters 78:2024-2027 (1997).

No. 12 Geritz SAH, Kisdi É, Meszéna G, Metz JAJ: Evolutionary Singular Strategies and the Adaptive Growth and Branching of the Evolutionary Tree. IIASA Working Paper WP-96-114 (1996). Evolutionary Ecology 12:35-57 (1998).

No. 13 Heino M, Metz JAJ, Kaitala V: Evolution of Mixed Maturation Strategies in Semelparous Life-Histories: The Crucial Role of Dimensionality of Feedback Environment. IIASA Working Paper WP-96-126 (1996). Philosophical Transactions of the Royal Society of London Series B 352:1647-1655 (1997).

No. 14 Dieckmann U: Can Adaptive Dynamics Invade? IIASA Working Paper WP-96-152 (1996). Trends in Ecology and Evolution 12:128-131 (1997).

No. 15 Meszéna G, Czibula I, Geritz SAH: Adaptive Dynamics in a 2-Patch Environment: A Simple Model for Allopatric and Parapatric Speciation. IIASA Interim Report IR-97-001 (1997). Journal of Biological Systems 5:265-284 (1997).

No. 16 Heino M, Metz JAJ, Kaitala V: The Enigma of Frequency-Dependent Selection. IIASA Interim Report IR97-061 (1997). Trends in Ecology and Evolution 13:367-370 (1998).

No. 17 Heino M: Management of Evolving Fish Stocks. IIASA Interim Report IR-97-062 (1997). Canadian Journal of Fisheries and Aquatic Sciences 55:1971-1982 (1998).

No. 18 Heino M: Evolution of Mixed Reproductive Strategies in Simple Life-History Models. IIASA Interim Report IR-97063 (1997).

No. 19 Geritz SAH, van der Meijden E, Metz JAJ: Evolutionary Dynamics of Seed Size and Seedling Competitive Ability. IIASA Interim Report IR-97-071 (1997). Theoretical Population Biology 55:324-343 (1999).

No. 20 Galis F, Metz JAJ: Why Are There So Many Cichlid Species? On the Interplay of Speciation and Adaptive Radiation. IIASA Interim Report IR-97-072 (1997). Trends in Ecology and Evolution 13:1-2 (1998). 
No. 21 Boerlijst MC, Nowak MA, Sigmund K: Equal Pay for all Prisoners/ The Logic of Contrition. IIASA Interim Report IR-97-073 (1997). American Mathematical Society Monthly 104:303-307 (1997). Journal of Theoretical Biology 185:281-293 (1997).

No. 22 Law R, Dieckmann U: Symbiosis Without Mutualism and the Merger of Lineages in Evolution. IIASA Interim Report IR-97-074 (1997). Proceedings of the Royal Society of London Series B 265:1245-1253 (1998).

No. 23 Klinkhamer PGL, de Jong TJ, Metz JAJ: Sex and Size in Cosexual Plants. IIASA Interim Report IR-97-078 (1997). Trends in Ecology and Evolution 12:260-265 (1997).

No. 24 Fontana W, Schuster P: Shaping Space: The Possible and the Attainable in RNA Genotype-Phenotype Mapping. IIASA Interim Report IR-98-004 (1998). Journal of Theoretical Biology 194:491-515 (1998).

No. 25 Kisdi É, Geritz SAH: Adaptive Dynamics in Allele Space: Evolution of Genetic Polymorphism by Small Mutations in a Heterogeneous Environment. IIASA Interim Report IR-98-038 (1998). Evolution 53:993-1008 (1999).

No. 26 Fontana W, Schuster P: Continuity in Evolution: On the Nature of Transitions. IIASA Interim Report IR-98-039 (1998). Science 280:1451-1455 (1998).

No. 27 Nowak MA, Sigmund K: Evolution of Indirect Reciprocity by Image Scoring/ The Dynamics of Indirect Reciprocity. IIASA Interim Report IR-98-040 (1998). Nature 393:573-577 (1998). Journal of Theoretical Biology 194:561574 (1998).

No. 28 Kisdi É: Evolutionary Branching Under Asymmetric Competition. IIASA Interim Report IR-98-045 (1998). Journal of Theoretical Biology 197:149-162 (1999).

No. 29 Berger U: Best Response Adaptation for Role Games. IIASA Interim Report IR-98-086 (1998).

No. 30 van Dooren TJM: The Evolutionary Ecology of Dominance-Recessivity. IIASA Interim Report IR-98-096 (1998). Journal of Theoretical Biology 198:519-532 (1999).

No. 31 Dieckmann U, O'Hara B, Weisser W: The Evolutionary Ecology of Dispersal. IIASA Interim Report IR-98-108 (1998). Trends in Ecology and Evolution 14:88-90 (1999).

No. 32 Sigmund K: Complex Adaptive Systems and the Evolution of Reciprocation. IIASA Interim Report IR-98-100 (1998). Ecosystems 1:444-448 (1998).

No. 33 Posch M, Pichler A, Sigmund K: The Efficiency of Adapting Aspiration Levels. IIASA Interim Report IR-98103 (1998). Proceedings of the Royal Society London Series B 266:1427-1435 (1999).

No. 34 Mathias A, Kisdi É: Evolutionary Branching and Coexistence of Germination Strategies. IIASA Interim Report IR-99-014 (1999).

No. 35 Dieckmann U, Doebeli M: On the Origin of Species by Sympatric Speciation. IIASA Interim Report IR-99-013 (1999). Nature 400:354-357 (1999).

No. 36 Metz JAJ, Gyllenberg M: How Should We Define Fitness in Structured Metapopulation Models? Including an Application to the Calculation of Evolutionarily Stable Dispersal Strategies. IIASA Interim Report IR-99-019 (1999). Proceedings of the Royal Society of London Series B 268:499508 (2001)
No. 37 Gyllenberg M, Metz JAJ: On Fitness in Structured Metapopulations. IIASA Interim Report IR-99-037 (1999). Journal of Mathematical Biology 43:545-560 (2001).

No. 38 Meszéna G, Metz JAJ: Species Diversity and Population Regulation: The Importance of Environmental Feedback Dimensionality. IIASA Interim Report IR-99-045 (1999).

No. 39 Kisdi É, Geritz SAH: Evolutionary Branching and Sympatric Speciation in Diploid Populations. IIASA Interim Report IR-99-048 (1999).

No. 40 Ylikarjula J, Heino M, Dieckmann U: Ecology and Adaptation of Stunted Growth in Fish. IIASA Interim Report IR-99-050 (1999). Evolutionary Ecology 13:433-453 (1999).

No. 41 Nowak MA, Sigmund K: Games on Grids. IIASA Interim Report IR-99-038 (1999). Dieckmann U, Law R, Metz JAJ (eds): The Geometry of Ecological Interactions: Simplifying Spatial Complexity, Cambridge University Press, Cambridge, UK, pp. 135-150 (2000).

No. 42 Ferrière R, Michod RE: Wave Patterns in Spatial Games and the Evolution of Cooperation. IIASA Interim Report IR-99-041 (1999). Dieckmann U, Law R, Metz JAJ (eds): The Geometry of Ecological Interactions: Simplifying Spatial Complexity, Cambridge University Press, Cambridge, UK, pp. 318-332 (2000).

No. 43 Kisdi É, Jacobs FJA, Geritz SAH: Red Queen Evolution by Cycles of Evolutionary Branching and Extinction. IIASA Interim Report IR-00-030 (2000). Selection 2:161$176(2001)$.

No. 44 Meszéna G, Kisdi É, Dieckmann U, Geritz SAH, Metz JAJ: Evolutionary Optimisation Models and Matrix Games in the Unified Perspective of Adaptive Dynamics. IIASA Interim Report IR-00-039 (2000). Selection 2:193-210 (2001).

No. 45 Parvinen K, Dieckmann U, Gyllenberg M, Metz JAJ: Evolution of Dispersal in Metapopulations with Local Density Dependence and Demographic Stochasticity. IIASA Interim Report IR-00-035 (2000). Journal of Evolutionary Biology 16:143-153 (2003).

No. 46 Doebeli M, Dieckmann U: Evolutionary Branching and Sympatric Speciation Caused by Different Types of Ecological Interactions. IIASA Interim Report IR-00-040 (2000). The American Naturalist 156:S77-S101 (2000).

No. 47 Heino M, Hanski I: Evolution of Migration Rate in a Spatially Realistic Metapopulation Model. IIASA Interim Report IR-00-044 (2000). The American Naturalist 157:495$511(2001)$.

No. 48 Gyllenberg M, Parvinen K, Dieckmann U: Evolutionary Suicide and Evolution of Dispersal in Structured Metapopulations. IIASA Interim Report IR-00-056 (2000). Journal of Mathematical Biology 45:79-105 (2002).

No. 49 van Dooren TJM: The Evolutionary Dynamics of Direct Phenotypic Overdominance: Emergence Possible, Loss Probable. IIASA Interim Report IR-00-048 (2000). Evolution 54:1899-1914 (2000).

No. 50 Nowak MA, Page KM, Sigmund K: Fairness Versus Reason in the Ultimatum Game. IIASA Interim Report IR00-57 (2000). Science 289:1773-1775 (2000).

No. 51 de Feo O, Ferrière R: Bifurcation Analysis of Population Invasion: On-Off Intermittency and Basin Riddling. IIASA Interim Report IR-00-074 (2000). International Journal of Bifurcation and Chaos 10:443-452 (2000). 
No. 52 Heino M, Laaka-Lindberg S: Clonal Dynamics and Evolution of Dormancy in the Leafy Hepatic Lophozia Silvicola. IIASA Interim Report IR-01-018 (2001). Oikos 94:525-532 (2001).

No. 53 Sigmund K, Hauert C, Nowak MA: Reward and Punishment in Minigames. IIASA Interim Report IR-01-031 (2001). Proceedings of the National Academy of Sciences of the USA 98:10757-10762 (2001).

No. 54 Hauert C, De Monte S, Sigmund K, Hofbauer J: Oscillations in Optional Public Good Games. IIASA Interim Report IR-01-036 (2001).

No. 55 Ferrière R, Le Galliard J: Invasion Fitness and Adaptive Dynamics in Spatial Population Models. IIASA Interim Report IR-01-043 (2001). Clobert J, Dhondt A, Danchin E, Nichols J (eds): Dispersal, Oxford University Press, pp. 57-79 (2001).

No. 56 de Mazancourt C, Loreau M, Dieckmann U: Can the Evolution of Plant Defense Lead to Plant-Herbivore Mutualism? IIASA Interim Report IR-01-053 (2001). The American Naturalist 158:109-123 (2001).

No. 57 Claessen D, Dieckmann U: Ontogenetic Niche Shifts and Evolutionary Branching in Size-Structured Populations. IIASA Interim Report IR-01-056 (2001). Evolutionary Ecology Research 4:189-217 (2002).

No. 58 Brandt H: Correlation Analysis of Fitness Landscapes. IIASA Interim Report IR-01-058 (2001).

No. 59 Dieckmann U: Adaptive Dynamics of Pathogen-Host Interacations. IIASA Interim Report IR-02-007 (2002). Dieckmann U, Metz JAJ, Sabelis MW, Sigmund K (eds): Adaptive Dynamics of Infectious Diseases: In Pursuit of Virulence Management, Cambridge University Press, Cambridge, UK, pp. 39-59 (2002).

No. 60 Nowak MA, Sigmund K: Super- and Coinfection: The Two Extremes. IIASA Interim Report IR-02-008 (2002). Dieckmann U, Metz JAJ, Sabelis MW, Sigmund K (eds): Adaptive Dynamics of Infectious Diseases: In Pursuit of Virulence Management, Cambridge University Press, Cambridge, UK, pp. 124-137 (2002).

No. 61 Sabelis MW, Metz JAJ: Evolution Management: Taking Stock - Relating Theory to Experiment. IIASA Interim Report IR-02-009 (2002). Dieckmann U, Metz JAJ, Sabelis MW, Sigmund K (eds): Adaptive Dynamics of Infectious Diseases: In Pursuit of Virulence Management, Cambridge University Press, Cambridge, UK, pp. 379-398 (2002).

No. 62 Cheptou P, Dieckmann U: The Evolution of SelfFertilization in Density-Regulated Populations . IIASA Interim Report IR-02-024 (2002). Proceedings of the Royal Society of London Series B 269:1177-1186(2002).

No. 63 Bürger R: Additive Genetic Variation Under Intraspecific Competition and Stabilizing Selection: A Two-Locus Study. IIASA Interim Report IR-02-013 (2002). Theoretical Population Biology 61:197-213 (2002).

No. 64 Hauert C, De Monte S, Hofbauer J, Sigmund K: Volunteering as Red Queen Mechanism for Co-operation in Public Goods Games. IIASA Interim Report IR-02-041 (2002). Science 296:1129-1132 (2002).

No. 65 Dercole F, Ferrière R, Rinaldi S: Ecological Bistability and Evolutionary Reversals under Asymmetrical Competition. IIASA Interim Report IR-02-053 (2002). Evolution 56:1081-1090 (2002).
No. 66 Dercole F, Rinaldi S: Evolution of Cannibalistic Traits: Scenarios Derived from Adaptive Dynamics. IIASA Interim Report IR-02-054 (2002). Theoretical Population Biology 62:365-374 (2002).

No. 67 Bürger R, Gimelfarb A: Fluctuating Environments and the Role of Mutation in Maintaining Quantitative Genetic Variation. IIASA Interim Report IR-02-058 (2002). Genetical Research 80:31-46 (2002).

No. 68 Bürger R: On a Genetic Model of Intraspecific Competition and Stabilizing Selection. IIASA Interim Report IR02-062 (2002). Amer. Natur. 160:661-682 (2002).

No. 69 Doebeli M, Dieckmann U: Speciation Along Environmental Gradients. IIASA Interim Report IR-02-079 (2002). Nature 421:259-264 (2003).

No. 70 Dercole F, Irisson J, Rinaldi S: Bifurcation Analysis of a Prey-Predator Coevolution Model. IIASA Interim Report IR-02-078 (2002). SIAM Journal on Applied Mathematics 63:1378-1391 (2003).

No. 71 Le Galliard J, Ferrière R, Dieckmann U: The Adaptive Dynamics of Altruism in Spatially Heterogeneous Populations. IIASA Interim Report IR-03-006 (2003). Evolution 57:1-17 (2003).

No. 72 Taborsky B, Dieckmann U, Heino M: Unexpected Discontinuities in Life-History Evolution under SizeDependent Mortality. IIASA Interim Report IR-03-004 (2003). Proceedings of the Royal Society of London Series B 270:713-721 (2003).

No. 73 Gardmark A, Dieckmann U, Lundberg P: LifeHistory Evolution in Harvested Populations: The Role of Natural Predation. IIASA Interim Report IR-03-008 (2003). Evolutionary Ecology Research 5:239-257 (2003).

No. 74 Mizera F, Meszéna G: Spatial Niche Packing, Character Displacement and Adaptive Speciation Along an Environmental Gradient. IIASA Interim Report IR-03-062 (2003). Evolutionary Ecology Research 5:363-382 (2003).

No. 75 Dercole F: Remarks on Branching-Extinction Evolutionary Cycles. IIASA Interim Report IR-03-077 (2003). Journal of Mathematical Biology 47:569-580 (2003).

No. 76 Hofbauer J, Sigmund K: Evolutionary Game Dynamics. IIASA Interim Report IR-03-078 (2003). Bulletin of the American Mathematical Society 40:479-519 (2003).

No. 77 Ernande B, Dieckmann U, Heino M: Adaptive Changes in Harvested Populations: Plasticity and Evolution of Age and Size at Maturation. IIASA Interim Report IR03-058 (2003). Proceedings of the Royal Society of London Series B-Biological Sciences 271:415-423 (2004).

No. 78 Hanski I, Heino M: Metapopulation-Level Adaptation of Insect Host Plant Preference and Extinction-Colonization Dynamics in Heterogeneous Landscapes. IIASA Interim Report IR-03-028 (2003). Theoretical Population Biology 63:309-338 (2003).

No. 79 van Doorn G, Dieckmann U, Weissing FJ: Sympatric Speciation by Sexual Selection: A Critical Re-Evaluation. IIASA Interim Report IR-04-003 (2004). American Naturalist 163:709-725 (2004).

No. 80 Egas M, Dieckmann U, Sabelis MW: Evolution Restricts the Coexistence of Specialists and Generalists - the Role of Trade-off Structure. IIASA Interim Report IR-04-004 (2004). American Naturalist 163:518-531 (2004). 
No. 81 Ernande B, Dieckmann U: The Evolution of Phenotypic Plasticity in Spatially Structured Environments: Implications of Intraspecific Competition, Plasticity Costs, and Environmental Characteristics. IIASA Interim Report IR-04-006 (2004). Journal of Evolutionary Biology 17:613-628 (2004).

No. 82 Cressman R, Hofbauer J: Measure Dynamics on a One-Dimensional Continuous Trait Space: Theoretical Foundations for Adaptive Dynamics. IIASA Interim Report IR04-016 (2004).

No. 83 Cressman R: Dynamic Stability of the Replicator Equation with Continuous Strategy Space. IIASA Interim Report IR-04-017 (2004).

No. 84 Ravigné V, Olivieri I, Dieckmann U: Implications of Habitat Choice for Protected Polymorphisms. IIASA Interim Report IR-04-005 (2004). Evolutionary Ecology Research 6:125-145 (2004).

No. 85 Nowak MA, Sigmund K: Evolutionary Dynamics of Biological Games. IIASA Interim Report IR-04-013 (2004). Science 303:793-799 (2004).

No. 86 Vukics A, Asbóth J, Meszéna G: Speciation in Multidimensional Evolutionary Space. IIASA Interim Report IR-04-028 (2004). Physical Review 68:041-903 (2003).

No. 87 de Mazancourt C, Dieckmann U: Trade-off Geometries and Frequency-dependent Selection. IIASA Interim Report IR-04-039 (2004). American Naturalist 164:765-778 (2004).

No. 88 Cadet CR, Metz JAJ, Klinkhamer PGL: Size and the Not-So-Single Sex: Disentangling the Effects of Size on Sex Allocation. IIASA Interim Report IR-04-084 (2004). American Naturalist 164:779-792 (2004).

No. 89 Rueffler C, van Dooren TJM, Metz JAJ: Adaptive Walks on Changing Landscapes: Levins' Approach Extended. IIASA Interim Report IR-04-083 (2004). Theoretical Population Biology 65:165-178 (2004).

No. 90 de Mazancourt C, Loreau M, Dieckmann U: Understanding Mutualism When There is Adaptation to the Partner. IIASA Interim Report IR-05-016 (2005). Journal of Ecology 93:305-314 (2005).

No. 91 Dieckmann U, Doebeli M: Pluralism in Evolutionary Theory. IIASA Interim Report IR-05-017 (2005). Journal of Evolutionary Biology 18:1209-1213 (2005).

No. 92 Doebeli M, Dieckmann U, Metz JAJ, Tautz D: What We Have Also Learned: Adaptive Speciation is Theoretically Plausible. IIASA Interim Report IR-05-018 (2005). Evolution 59:691-695 (2005).

No. 93 Egas M, Sabelis MW, Dieckmann U: Evolution of Specialization and Ecological Character Displacement of Herbivores Along a Gradient of Plant Quality. IIASA Interim Report IR-05-019 (2005). Evolution 59:507-520 (2005).

No. 94 Le Galliard J, Ferrière R, Dieckmann U: Adaptive Evolution of Social Traits: Origin, Trajectories, and Correlations of Altruism and Mobility. IIASA Interim Report IR05-020 (2005). American Naturalist 165:206-224 (2005).

No. 95 Doebeli M, Dieckmann U: Adaptive Dynamics as a Mathematical Tool for Studying the Ecology of Speciation Processes. IIASA Interim Report IR-05-022 (2005). Journal of Evolutionary Biology 18:1194-1200 (2005).

No. 96 Brandt H, Sigmund K: The Logic of Reprobation: Assessment and Action Rules for Indirect Reciprocity. IIASA Interim Report IR-04-085 (2004). Journal of Theoretical Biology 231:475-486 (2004).
No. 97 Hauert C, Haiden N, Sigmund K: The Dynamics of Public Goods. IIASA Interim Report IR-04-086 (2004). Discrete and Continuous Dynamical Systems - Series B 4:575587 (2004).

No. 98 Meszéna G, Gyllenberg M, Jacobs FJA, Metz JAJ: Link Between Population Dynamics and Dynamics of Darwinian Evolution. IIASA Interim Report IR-05-026 (2005). Physical Review Letters 95:Article 078105 (2005).

No. 99 Meszéna G: Adaptive Dynamics: The Continuity Argument. IIASA Interim Report IR-05-032 (2005).

No. 100 Brännström NA, Dieckmann U: Evolutionary Dynamics of Altruism and Cheating Among Social Amoebas. IIASA Interim Report IR-05-039 (2005). Proceedings of the Royal Society London Series B 272:1609-1616 (2005).

No. 101 Meszéna G, Gyllenberg M, Pasztor L, Metz JAJ: Competitive Exclusion and Limiting Similarity: A Unified Theory. IIASA Interim Report IR-05-040 (2005).

No. 102 Szabo P, Meszéna G: Limiting Similarity Revisited. IIASA Interim Report IR-05-050 (2005).

No. 103 Krakauer DC, Sasaki A: The Greater than Two-Fold Cost of Integration for Retroviruses. IIASA Interim Report IR-05-069 (2005).

No. 104 Metz JAJ: Eight Personal Rules for Doing Science. IIASA Interim Report IR-05-073 (2005). Journal of Evolutionary Biology 18:1178-1181 (2005).

No. 105 Beltman JB, Metz JAJ: Speciation: More Likely Through a Genetic or Through a Learned Habitat Preference? IIASA Interim Report IR-05-072 (2005). Proceedings of the Royal Society of London Series B 272:1455-1463 (2005).

No. 106 Durinx M, Metz JAJ: Multi-type Branching Processes and Adaptive Dynamics of Structured Populations. IIASA Interim Report IR-05-074 (2005). Haccou P, Jager P, Vatutin V (eds): Branching Processes: Variation, Growth and Extinction of Populations, Cambridge University Press, Cambridge, UK, pp. 266-278 (2005).

No. 107 Brandt H, Sigmund K: The Good, the Bad and the Discriminator - Errors in Direct and Indirect Reciprocity. IIASA Interim Report IR-05-070 (2005). Journal of Theoretical Biology 239:183-194 (2006).

No. 108 Brandt H, Hauert C, Sigmund K: Punishing and Abstaining for Public Goods. IIASA Interim Report IR-05-071 (2005). Proceedings of the National Academy of Sciences of the United States of America 103:495-497 (2006).

No. 109 Ohtsuki A, Sasaki A: Epidemiology and DiseaseControl Under Gene-for-Gene Plant-Pathogen Interaction. IIASA Interim Report IR-05-068 (2005).

No. 110 Brandt H, Sigmund K: Indirect Reciprocity, ImageScoring, and Moral Hazard. IIASA Interim Report IR-05078 (2005). Proceedings of the National Academy of Sciences of the United States of America 102:2666-2670 (2005).

No. 111 Nowak MA, Sigmund K: Evolution of Indirect Reciprocity. IIASA Interim Report IR-05-079 (2005). Nature 437:1292-1298 (2005).

No. 112 Kamo M, Sasaki A: Evolution Towards Multi-Year Periodicity in Epidemics. IIASA Interim Report IR-05-080 (2005). Ecology Letters 8:378-385 (2005). 
No. 113 Dercole F, Ferrière R, Gragnani A, Rinaldi S: Coevolution of Slow-fast Populations: Evolutionary Sliding, Evolutionoary Pseudo-equilibria, and Complex Red Queen Dynamics. IIASA Interim Report IR-06-006 (2006). Proceedings of the Royal Society B 273:983-990 (2006).

No. 114 Dercole F: Border Collision Bifurcations in the Evolution of Mutualistic Interactions. IIASA Interim Report IR-05-083 (2005). International Journal of Bifurcation and Chaos 15:2179-2190 (2005).

No. 115 Dieckmann U, Heino M, Parvinen K: The Adaptive Dynamics of Function-Valued Traits. IIASA Interim Report IR-06-036 (2006). Journal of Theoretical Biology 241:370389 (2006).

No. 116 Dieckmann U, Metz JAJ: Surprising Evolutionary Predictions from Enhanced Ecological Realism. IIASA Interim Report IR-06-037 (2006). Theoretical Population Biology 69:263-281 (2006).

No. 117 Dieckmann U, Brännström NA, HilleRisLambers R, Ito H: The Adaptive Dynamics of Community Structure. IIASA Interim Report IR-06-038 (2006). Takeuchi Y, Iwasa Y, Sato K (eds): Mathematics for Ecology and Environmental Sciences, Springer, Berlin Heidelberg, pp. 145-177 (2007).

No. 118 Gardmark A, Dieckmann U: Disparate Maturation Adaptations to Size-dependent Mortality. IIASA Interim Report IR-06-039 (2006). Proceedings of the Royal Society London Series B 273:2185-2192(2006).

No. 119 van Doorn G, Dieckmann U: The Long-term Evolution of Multi-locus Traits Under Frequency-dependent Disruptive Selection. IIASA Interim Report IR-06-041 (2006). Evolution 60:2226-2238 (2006).

No. 120 Doebeli M, Blok HJ, Leimar O, Dieckmann U: Multimodal Pattern Formation in Phenotype Distributions of Sexual Populations. IIASA Interim Report IR-06-046 (2006). Proceedings of the Royal Society London Series B 274:347357 (2007).

No. 121 Dunlop ES, Shuter BJ, Dieckmann U: The Demographic and Evolutionary Consequences of Selective Mortality: Predictions from an Eco-genetic Model of the Smallmouth Bass. IIASA Interim Report IR-06-060 (2006). Transactions of the American Fisheries Society 136:749-765 (2007).

No. 122 Metz JAJ: Fitness. IIASA Interim Report IR-06061 (2006).
No. 123 Brandt H, Ohtsuki H, Iwasa Y, Sigmund K: A Survey on Indirect Reciprocity. IIASA Interim Report IR-06-065 (2006). Takeuchi Y, Iwasa Y, Sato K (eds): Mathematics for Ecology and Environmental Sciences, Springer, Berlin Heidelberg, pp. 21-51 (2007).

No. 124 Dercole F, Loiacono D, Rinaldi S: Synchronization in Ecological Networks: A Byproduct of Darwinian Evolution? IIASA Interim Report IR-06-068 (2006).

No. 125 Dercole F, Dieckmann U, Obersteiner M, Rinaldi S: Adaptive Dynamics and Technological Change. IIASA Interim Report IR-06-070 (2006).

No. 126 Rueffler C, van Dooren TJM, Metz JAJ: The Evolution of Resource Specialization Through FrequencyDependent and Frequency-Independent Mechanisms. IIASA Interim Report IR-06-073 (2006). American Naturalist 167:81-93 (2006).

No. 127 Rueffler C, Egas M, Metz JAJ: Evolutionary Predictions Should be Based on Individual Traits. IIASA Interim Report IR-06-074 (2006). American Naturalist 168:148-162 (2006).

No. 128 Kamo M, Sasaki A, Boots M: The Role of Trade-Off Shapes in the Evolution of Virulence in Spatial Host-Parasite Interactions: An Approximate Analytical Approach . IIASA Interim Report IR-06-075 (2006).

No. 129 Boots M, Kamo M, Sasaki A: The Implications of Spatial Structure Within Populations to the Evolution of Parasites. IIASA Interim Report IR-06-078 (2006).

No. 130 Andreasen V, Sasaki A: Shaping the Phylogenetic Tree of Influenza by Cross-Immunity. IIASA Interim Report IR-06-079 (2006).

No. 131 Rueffler C, van Dooren TJM, Metz JAJ: The Interplay Between Behavior and Morphology in the Evolutionary Dynamics of Resource Specialization. IIASA Interim Report IR-06-082 (2006). American Naturalist 169:E34-E52 (2007).

No. 132 Rueffler C, van Dooren TJM, Metz JAJ: The Evolution of Simple Life-Histories: Steps Towards a Classification. IIASA Interim Report IR-06-083 (2006).

No. 133 Durinx M, Metz JAJ, Meszéna G: Adaptive Dynamics for Physiologically Structured Population Models. IIASA Interim Report IR-07-027 (2007).

No. 134 Ito H, Dieckmann U: A New Mechanism for Recurrent Adaptive Radiations. IIASA Interim Report IR-07-048 (2007). American Naturalist 170:E96-E111 (2007).

Issues of the IIASA Studies in Adaptive Dynamics series can be obtained at www.iiasa.ac.at/Research/EEP/Series.html or by writing to eep@iiasa.ac.at. 


\section{Contents}

Abstract 


\title{
A new mechanism for recurrent adaptive radiations
}

\author{
Hiroshi C. Ito ${ }^{1,2}$ and Ulf Dieckmann ${ }^{2,3}$
}

1 The Graduate School of Arts and Sciences, University of Tokyo, 3-8-1 Komaba, Meguro-ku, Tokyo 153-8902, Japan

2 Evolution and Ecology Program, International Institute for Applied Systems Analysis, Schlossplatz 1, A-2361 Laxenburg, Austria

3 Section Theoretical Biology, Institute of Biology, Leiden University, Kaiserstraat 63, NL-2311 GP Leiden, The Netherlands

Author for correspondence: Hiroshi C. Ito

E-mail addresses: H. C. Ito, itoh9@dolphin.c.u-tokyo.ac.jp; U. Dieckmann, dieckmann@iiasa.ac.at

Keywords: adaptive radiation, extinction, frequency-dependent selection, evolutionary branching, taxon cycle

Online data and material: None

Color figures: fig. 1, fig. 3, fig. 4

Manuscript type: Article 


\begin{abstract}
Models of adaptive radiation through intraspecific competition have attracted mounting attention. Here we show how extending such models in a simple manner, by including a quantitative trait under weak directional selection, naturally leads to rich macroevolutionary patterns involving recurrent adaptive radiations and extinctions. Extensive tests demonstrate the robustness of this finding to a wide range of variations in model assumptions. In particular, recurrent adaptive radiations and extinctions readily unfold both for asexual and for sexual populations. Since the mechanisms driving the investigated processes of endogenous diversification result from generic geometric features of the underlying fitness landscapes - frequency-dependent disruptive selection in one trait and weak directional selection in another - the reported phenomena can be expected to occur in a wide variety of eco-evolutionary settings.
\end{abstract}




\section{Introduction}

Changes in biodiversity result from speciation and extinction (McKinney \& Drake 1998; Morris 1998) and may involve both endogenous and exogenous factors. Adaptive radiations driven by ecological interactions (Bush 1975; Schluter 1994, 2000; Feder et al. 1997; Grant 1998; Orr \& Smith 1998) are key endogenous processes crucial for understanding the generation of biodiversity.

Theoretical studies have consistently emphasized the importance of frequency-dependent selection for driving populations towards fitness minima at which selection turns disruptive and where adaptive radiations may thus occur (Rosenzweig 1978; Christiansen 1991; Brown \& Pavlovic 1992; Metz et al. 1992; Abrams et al. 1993; Metz et al. 1996; Geritz et al. 1997, 1998; Cohen et al. 1999). Such processes have been termed evolutionary branching, and the locations of evolutionarily attracting fitness minima in an organism's trait space are known as evolutionary branching points.

A plethora of studies have predicted evolutionary branching to occur in eco-evolutionary models of all fundamental types of ecological interaction (Doebeli \& Dieckmann 2000). In particular, evolutionary branching has been studied in the context of symmetric intraspecific competition (Metz et al. 1996; Doebeli 1996a, 1996b; Dieckmann \& Doebeli 1999), asymmetric intraspecific competition (Kisdi 1999; Doebeli \& Dieckmann 2000; Kisdi et al. 2001), interspecific competition (Law et al. 1997; Kisdi \& Geritz 2001), resource specialization (Meszéna et al. 1997; Geritz et al. 1998; Day 2000; Kisdi 2001; Schreiber \& Tobiason 2003; Egas et al. 2004, 2005), ontogenetic niche shifts (Claessen \& Dieckmann 2002), mixotrophy (Troost et al. 2005), phenotypic plasticity (Van Dooren \& Leimar 2003; Ernande \& Dieckmann 2004; Leimar 2005), dispersal evolution (Doebeli and Ruxton 1997; Johst et al. 1999; Parvinen 1999; Mathias et al. 2001; Parvinen \& Egas 2004), mutualism (Doebeli \& Dieckmann 2000; Law et al. 2001; Ferdy et al. 2002; Ferrière et al. 2002; Day \& Young 2004), emergent cooperation (Doebeli et al. 2004), predator-prey interactions (Brown \& Pavlovic 1992; Van der Laan \& Hogeweg 1995; Doebeli \& Dieckmann 2000; Bowers et al. 2003), cannibalism (Dercole 2003), host- 
parasite interactions (Boots \& Haraguchi 1999; Koella \& Doebeli 1999; Regoes et al. 2000; Gudelj et al. 2004), sex-ratio evolution (Metz et al. 1992; Reuter et al. 2004), evolution of selfing (Cheptou \& Mathias 2001; De Jong \& Geritz 2001), evolution of mating traits (Van Doorn et al. 2001, 2004), evolution of anisogamy (Maire et al. 2001), seed evolution (Geritz et al. 1999; Mathias \& Kisdi 2002), microbial cross-feeding (Doebeli 2002), prebiotic evolution (Meszéna \& Szathmáry 2001), resource competition among digital organisms (Chow et al. 2004), and evolutionary community assembly (Jansen \& Mulder 1999; Bonsall et al. 2004; Loeuille \& Loreau 2005). A recent review of findings in the related field of research on ecological character displacement (Brown \& Wilson 1956) has been provided by Dayan \& Simberloff (2005). The number of studies quoted above indicates the extraordinarily wide range of ecological settings for which disruptive frequency-dependent selection may naturally cause evolutionary diversification. Addressing the origin, maintenance, and loss of biological diversity, analyses of these evolutionary processes contribute to an improved understanding of some of the most fundamental questions in biology.

To date, most studies of evolutionary branching have focused on a single quantitative character. Necessary and sufficient analytical conditions for evolutionary branching to occur in more than onedimensional trait spaces have yet to be derived. Therefore, the question how evolution in an extra quantitative character may interfere with processes of evolutionary branching has not been analyzed systematically yet. Here we take a first step in this direction by considering the joint evolution of two quantitative characters, one of which is under frequency-dependent disruptive selection while the other is under weak directional selection. We explain how this simple extension of earlier models results in surprisingly rich macroevolutionary patterns, involving recurrent adaptive radiations and extinctions. We find macroevolution in which evolutionary branching repeatedly occurs in the character under disruptive selection, with some of the resultant lineages subsequently being excluded by lineage-level selection on the character under directional selection, causing persistent Red Queen coevolution (Van Valen 1973) at the community level. 
Complementing research into the ecological underpinnings of evolutionary diversification, another line of recent work has elucidated the circumstances under which the frequency-dependent disruptive selection pressures emerging at evolutionary branching points may overcome the genetic cohesion of sexually reproducing populations. Extending pioneering work by Maynard Smith (1966), Udovic (1980), and Felsenstein (1981), these studies have shown that, under some conditions, the degrees of reproductive isolation that can adaptively evolve at evolutionary branching points are sufficient even for sexual populations to split up under the pressure of disruptive selection (Dieckmann \& Doebeli 1999; Kisdi \& Geritz 1999; Kondrashov \& Kondrashov 1999). In this way, sexual populations may escape from being perpetually trapped at fitness minima. How easily and quickly such escapes must be expected to occur is a matter of much current investigation and debate (e.g., Matessi et al. 2001; Bolnick 2004; Doebeli \& Dieckmann 2005; Gavrilets 2005). Overviews are provided by Dieckmann et al. (2004) and Doebeli et al. (2005). While our study here is primarily concerned with patterns of phenotypic evolution in asexual populations, and therefore does not aspire to contribute to the more intricate controversies of contemporary speciation theory, an effort is nevertheless made below to illustrate how our results about recurrent radiations and extinctions are expected to carry over to sexual populations.

This article is structured as follows. The section "Model description" introduces models of bivariate evolution driven by intraspecific competition, and details their individual-based foundation, both for asexual and for sexual populations. The section "Results" presents our main findings about the macroevolutionary patterns of recurrent radiations and extinctions resulting in these models. This is accompanied by a series of tests that critically evaluate the robustness of our findings. The section "Discussion" reflects on the generality of the evolutionary phenomena reported here and concludes with appraising their relevance for understanding taxon cycles. 


\section{Model description}

Real populations invariably evolve in multidimensional trait spaces. It must therefore be assumed that a population that reaches an evolutionary branching point in one trait will still experience directional selection in at least one other trait. In order to investigate the evolutionary implications of such presumably very common settings, we extend a standard family of models originally derived by MacArthur (1972) and used by Roughgarden $(1974,1976)$ to investigate the evolutionary consequences of intra- and interspecific competition.

\section{One-dimensional model}

Following many earlier studies, we consider individuals characterized by a one-dimensional quantitative character $x$ that affects intraspecific competition (as, e.g., when beak size in birds determines the size of seeds they compete for). The per capita birth rate of individuals is assumed to be constant,

$$
b(x)=r,
$$

while their per capita death rate $d(x)$ depends on their trait value $x$ as well as on abundances of extant phenotypes $n_{j}$ for $j=1, . ., N$,

$$
d(x)=r \sum_{j} \alpha\left(x_{j}-x\right) n_{j} / K(x)
$$

Here

$$
K(x)=K_{0} \exp \left(-\frac{1}{2} x^{2} / \sigma_{K}^{2}\right)
$$

is the carrying capacity of phenotype $x$, given by a Gaussian function with variance $\sigma_{K}^{2}$, peaked at 0 . The function

$$
\alpha\left(x_{j}-x\right)=\exp \left(-\frac{1}{2}\left(x_{j}-x\right)^{2} / \sigma_{\alpha}^{2}\right)
$$

describes the strength of competition between phenotype $x$ and phenotype $x_{j}$; it is also Gaussian with variance $\sigma_{\alpha}^{2}$ and peaked at 0 . Accordingly, the strength of competition is maximal between identical 
phenotypes and monotonically declines with phenotypic distance. In the limit of infinite population size, the assumptions above imply Lotka-Volterra population dynamics, $\frac{d}{d t} n_{i}=\left[b\left(x_{i}\right)-d\left(x_{i}\right)\right] n_{i}$ for $i=1, . ., N$. To scale time, we assume $r=1$ without any loss of generality.

When the quantitative character $x$ is allowed to adapt through rare and small steps $x \rightarrow x^{\prime}$ from an initial value $x \neq 0$, the population first converges on the carrying capacity's maximum at $x=0$. For $\sigma_{\alpha}>\sigma_{K}$, this outcome is evolutionarily stable. By contrast, when competition is sufficiently focused on similar phenotypes, $\sigma_{\alpha}<\sigma_{K}$, the population at the carrying capacity's maximum experiences a fitness minimum, resulting in disruptive frequency-dependent selection. In this situation, rare phenotypes $x \neq 0$ gain more from diminished competition with the common phenotype at $x=0$ than they lose in terms of reduced carrying capacity. Accordingly, a gradually evolving monomorphic asexual population is expected to become dimorphic at the evolutionary branching point $x=0$ (Metz et al. 1992, 1996; Geritz et al. 1997, 1998).

Model (1), or slight variants thereof, have been investigated by many authors, including Christiansen \& Fenchel (1977), Christiansen \& Loeschcke (1980), Slatkin (1980), Case (1981), Seger (1985), Taper \& Case (1985), Vincent et al. (1993), Metz et al. (1996), Doebeli (1996b), Dieckmann \& Doebeli (1999), Drossel \& McKane (2000), Day (2000), Ackermann \& Doebeli (2004), and Doebeli et al. (2007).

\section{Two-dimensional model}

Model (1) is readily extended to a two-dimensional quantitative character $(x, y)$,

$$
\begin{gathered}
b(x, y)=r, \\
d(x, y)=r \sum_{j} \alpha\left(x_{j}-x, y_{j}-y\right) n_{j} / K(x, y), \\
K(x, y)=K_{0} \exp \left(-\frac{1}{2} x^{2} / \sigma_{K x}^{2}-\frac{1}{2} y^{2} / \sigma_{K y}^{2}\right),
\end{gathered}
$$




$$
\alpha\left(x_{j}-x, y_{j}-y\right)=\exp \left(-\frac{1}{2}\left(x_{j}-x\right)^{2} / \sigma_{\alpha x}^{2}-\frac{1}{2}\left(y_{j}-y\right)^{2} / \sigma_{\alpha y}^{2}\right) .
$$

For any fixed $y, x=0$ is an evolutionary branching point if $\sigma_{\alpha x}<\sigma_{K x}$. However, Vukics \& Meszéna (2003) argued that evolutionary branching in this two-dimensional model only occurs at $(x, y)=(0,0)$, since it is only at this point that directional selection pressures vanish for both $x$ and $y$. This conclusion indeed holds when speeds of evolution in the two traits are comparable. In this case, two phenotypes forming a narrow dimorphism in trait $x$ will typically be replaced by a mutant in trait $y$ that is superior to both of them. If we assume, by contrast, significantly slower evolution in trait $y$ than in trait $x$ (resulting either from a weaker fitness gradient or from smaller or rarer mutations), the emergence of a dimorphism in trait $x$ may lead to evolutionary diversification. As we will demonstrate in detail below, in the section "Results", evolutionary branching may then occur also away from $(x, y)=(0,0)$. The only requirement is that selection is frequency-dependent disruptive in one direction of trait space and weakly directional in the remaining direction. In the two-dimensional model (2), this situation arises for $\sigma_{\alpha x}<\sigma_{K x}$ and $\sigma_{K x} \ll \sigma_{K y}$. Under these conditions, evolutionary branching may occur along the entire line $x=0$.

\section{Simplified two-dimensional model}

Directional selection on $y$ can be due to any ecological interaction - including competition, predatorprey interaction, or mutualism - and can act on any morphological, physiological, life-history, or behavioral character $y$. A simple way of enhancing model (1) by introducing a fitness gradient in $y$ is given by

$$
\begin{aligned}
& b(x, y)=b(x)+b_{1} \cdot(y-\bar{y}), \\
& d(x, y)=d(x)+d_{1} \cdot(y-\bar{y}),
\end{aligned}
$$


where $b(x)$ and $d(x)$ are defined as in (1a) and (1b), $\bar{y}$ denotes the population average of trait value $y, \bar{y}=\sum_{j} y_{j} n_{j} / \sum_{j} n_{j}$, and $b_{1}$ and $d_{1}$ are constants.

In this model, any shift in the distribution of $y$ that changes $\bar{y}$ but not the differences $y-\bar{y}$ leaves the population's per capita birth and death rates unaffected, thus describing characters with demographic effects determined by relative trait differences rather than absolute trait values. This situation is typical for characters under frequency-dependent directional selection. Two key mechanisms implying such frequency dependence are trait-dependent density regulation and (conspecific or heterospecific) arms races. These may cancel the effects of directional evolution in a population's mean $\bar{y}$ through density adjustments or concomitant evolution, respectively.

In model (3), composed of (3a) and (3b), the strength of directional selection in $y$, measured by the fitness gradient in $y$, is given by

$$
G_{y}=\left.\frac{\partial[b(x, y)-d(x, y)]}{\partial y}\right|_{x=\bar{x}, y=\bar{y}}=b_{1}-d_{1} .
$$

The strength of disruptive selection in $x$ is measured by the second derivative of growth rate with respect to $x$. For a monomorphic population at $x=0$ this yields

$$
D_{x}=\left.\frac{\partial^{2}[b(x, y)-d(x, y)]}{\partial x^{2}}\right|_{x=\bar{x}=0, y=\bar{y}}=\sigma_{\alpha}^{-2}-\sigma_{K}^{-2} .
$$

Whenever $b(x, y)$ would become negative according to $(3 \mathrm{a})$, it is set to 0 . When mutations are rare and small, $b(x, y)$ is almost always positive: rare mutations keep population dynamics close to the equilibrium at which $b(x, y)$ is close to $d(x, y)>0$, and small mutations change these rates only slightly. Similarly, $d(x, y)$ in (3b) is set to 0 whenever it would become negative. How (3a) and (3b) can be derived from arbitrary forms of $b(x, y)$ and $d(x, y)$ is explained in Appendix A. 
Like in the full two-dimensional model, evolutionary branching in the simplified model may occur along the line $x=0$ when $G_{y}$ is small compared to $D_{x}$. After such evolutionary branching, the resultant pair of lineages will continuously evolve in $y$ under the constant, directional selection pressure $G_{y}$. In the course of this evolution, which will usually be accompanied by further divergence in $x$, asymmetries in values of $y$ naturally build up between the lineages, eventually destroying their coexistence. Thus, once evolutionary branching has occurred, extinction is inevitable. Our analyses below examine how this intricate interplay between radiations and extinctions unfolds.

\section{Asexual individual-based models}

To allow for asexual evolution, we assume that, with a small probability $\mu$, a birth event involves a mutation. In the one-dimensional model, mutant trait values $x^{\prime}$ are drawn from a univariate Gaussian distribution with variance $\sigma_{\mu}^{2}$, peaked at the parental phenotype $x$. In the two-dimensional models, mutant trait values $\left(x^{\prime}, y^{\prime}\right)$ are drawn from a bivariate covariance-free Gaussian distribution with variances $\sigma_{\mu x}^{2}$ and $\sigma_{\mu y}^{2}$, peaked at the parental phenotype $(x, y)$.

The individual- and event-based asexual models defined by combining the mutation process specified above with the ecological rates specified for models (1) to (3) are implemented using Gillespie's minimal process method for homogeneous Markov processes in continuous time (Gillespie 1976; see also Dieckmann 1994; Dieckmann et al. 1995). Moreover, to enable the fast calculation of long-term evolutionary dynamics, asexual evolution is approximated by assuming low rates of mutation (our numerical analyses have confirmed that this approximation does not affect the dynamics in any qualitative way). Implementation details are provided in Appendix B. 


\section{Sexual individual-based models}

Sexual evolution is incorporated into models (1) to (3) by considering male and female individuals, diploid inheritance, and quantitative characters coded for by multilocus genetics. There are various ways of modeling mating systems, including those directly based on ecological traits or, alternatively, on other mating traits. Since conditions for evolutionary branching have been shown to be more restrictive for the latter setting compared to the former (Dieckmann \& Doebeli 1999, 2004), we used the latter setting, with a mating system based on additional mating characters, to check the robustness of results obtained for the asexual model under the most challenging conditions.

Specifically, $L_{x}$ and $L_{y}$ loci are considered for $x$ and $y$, respectively, with integer allelic values, and with the value of the coded quantitative characters being given by the average of allelic values across loci. To allow some flexibility with regard to recombination, $C_{x}$ and $C_{y}$ linkage clusters are considered for $x$ and $y$ : while there is no recombination within such a cluster, recombination between them is free. Females produce offspring individuals at rate $2 b_{i}$, where $b_{i}$ denotes the per capita birth rate of a female $i$ as specified by models (1) to (3). (The factor 2 arises in this correspondence since males do not bear offspring.) A female individual $i$ chooses a male partner $j$ for mating with probability

$$
\tilde{P}_{i j}=P_{i j} b_{j} / \sum_{k} P_{i k} b_{k},
$$

where $b_{j}$ denotes the per capita birth rate of male $j$ and the sum extends over all males in the population. The mating probability $P_{i j}$ depends on a display character $m_{j}$ in male $j$ (e.g., pattern, color, song, dance, or gift) and on a corresponding preference character $p_{i}$ in female $i$. These characters involve $L_{m}=L_{p}$ loci, which are organized into $C_{m}$ and $C_{p}$ linkage clusters. As for $x$ and $y$, all allelic values are integers. The quantitative characters $m_{j}$ and $p_{i}$ are multivariate, with each of their compo- 
nents $m_{i 1}, \ldots, m_{i L_{m}}$ and $p_{i 1}, \ldots, p_{i L_{p}}$ being determined by averaging the two allelic values at the corresponding pair of homologous loci. The mating probability $P_{i j}$ is then given by

$$
P_{i j}=\exp \left(-\frac{1}{2} d_{i j}^{2} / \sigma_{m}^{2}\right)
$$

with

$$
d_{i j}^{2}=\left(p_{i}-m_{j}\right)^{2}=\sum_{k=1}^{L_{m}}\left(p_{i k}-m_{j k}\right)^{2},
$$

so that $d_{i j}$ measures the (Euclidian) distance between the preference of female $i$ and the display of male $j$. Mating probabilities are thus increasing with the similarity between a male's display character and a female's preference character, with $\sigma_{m}$ measuring the strength of female choosiness. Allelic mutations that increase or decrease allelic values (which happens with equal probability) occur with per locus probabilities of $\mu_{x}, \mu_{y}, \mu_{m}, \mu_{p}$ at the time offspring is formed. The sex of offspring is assigned at random, assuming an even primary sex ratio.

The individual- and event-based sexual models defined by combining the process of sexual reproduction specified above with the ecological rates specified for models (1) to (3) are again implemented using Gillespie's minimal process method. Implementation details are provided in Appendix C.

\section{Results}

We first demonstrate the typical evolutionary dynamics of recurrent adaptive radiations and extinctions observed in the simplified two-dimensional model with asexual reproduction, before showing that the same dynamics arise in the full two-dimensional model and in models with sexual reproduction.

\section{Recurrent radiations and extinctions}

Our results reveal that the inclusion of directional selection in trait $y$ qualitatively alters the observed macroevolutionary dynamics. Three different regimes can be distinguished. 
First, in the complete absence of directional selection in trait $y, G_{y}=0$, convergence to, and evolutionary branching at, $x=0$ is followed by further processes of evolutionary branching, establishing a static configuration of lineages (figure 1a). Owing to frequency-dependent selection, the current fitness landscape is contingent on the resident phenotypes. As long as the evolving resident population remains monomorphic, a single evolutionary branching point exists, $x_{b 1}=0$. Once the resident population has become dimorphic, this evolutionary branching point is replaced by two others, $x_{b 2}^{(1)}=-x_{b 2}^{(2)} \neq 0$, at which the resident population can become trimorphic and quadrumorphic. For finite populations, this process of sequential evolutionary branching results in no more than a finite number of lineages, as the residual strength of disruptive selection in trait $x$ diminishes with each branching.

Second, when directional selection in trait $y$ is very strong, evolution in trait $y$ is so swift that it prevents diversification in trait $x$. After the population has converged to $x=0$, it thus merely keeps evolving along this line, in response to the directional selection pressure $G_{y}$ (results not shown).

Third, when directional selection in trait $y$ is finite and sufficiently weak, something very different happens: the initial branching in trait $x$ is followed by a pattern of recurrent adaptive radiations and extinctions (figure 1b). By comparing figures $1 \mathrm{a}$ and $1 \mathrm{~b}$, we can appreciate how the weak directional selection in trait $y$ changes the macroevolutionary pattern. Instead of the static polymorphism resulting for the one-dimensional model - or, equivalently, for $G_{y}=0$ - the two-dimensional model gives rise to incessant macroevolution, resulting in a more intricate, dynamically regenerating evolutionary tree.

The evolutionary mechanism responsible for the observed pattern of recurrent adaptive radiations and extinctions can be understood as follows. Right after evolutionary branching, stochastic effects resulting from mutations, as well as from the demography of finite populations - cause the spontaneous breaking of the initial symmetry between the two diverging lineages. In particular, the population sizes of the two lineages will never be exactly equal. Since the more abundant lineage can evolve faster, it 
will move ahead in the race of responding to the weak directional selection pressure in trait $y$, thus increasing its relative fitness in terms of trait $y$. We refer to the more abundant, leading lineage as the anterior lineage and to the less abundant, trailing lineage as the posterior lineage. The evolutionary interplay between these two lineages causes the asymmetry in their population sizes to grow and their trait values $y$ to diverge. This positive feedback continues until the posterior lineage goes extinct (figure 1c). The positive feedback may be intensified by an additional effect: once the anterior lineage becomes sufficiently dominant, it experiences so little competition from the posterior lineage that it reverses its direction of gradual evolution in trait $x$, thus pushing the posterior lineage towards lower carrying capacity and accelerating its demise (figure $1 \mathrm{~b}$ ).

\section{Robustness: niche widths}

We examined how these basic macroevolutionary patterns and mechanisms extend to ecological settings involving broader fundamental niches. In our models, this corresponds to widening the carrying capacity function $K$ with respect to trait $x$. Without directional selection in trait $y$, a large value of $\sigma_{K}$ (measured relative to the range of competition, $\sigma_{\alpha}$ ) accelerates evolutionary branching and enables

a larger number of coexisting lineages (figure 1d). Similarly, when directional selection in trait $y$ is weak but finite, a wide fundamental niche permits secondary and tertiary evolutionary branching before the first extinction occurs. This results in a larger number of coexisting lineages becoming established and reestablished through the dynamic balance between adaptive radiation and extinction (figure 1e).

While the evolutionary mechanisms underlying these patterns are the same as described above, the salient evolutionary interplay now occurs between several (instead of just two) lineages. Accordingly, we must interpret the macroevolutionary dynamics at the community level, in terms of anterior and posterior groups of lineages (figure 1f). Yet another asymmetry-inducing evolutionary mechanism thus becomes important: anterior lineages are not only exhibiting faster gradual evolution, but also faster 
adaptive radiations. This effect induces the explosive radiations of anterior lineages, accompanied by mass extinctions in all other lineages (see, e.g., the momentous radiation after $t=0.3 \times 10^{6}$ in figures $1 \mathrm{e}$ and 1f). When advantageous mutations in trait $y$ are made large but rare, the described effect inevitably leads to evolutionary dynamics of 'punctuated equilibrium' type (Gould \& Eldredge 1977).

\section{Robustness: selection strengths}

How are these macroevolutionary patterns quantitatively affected by the strength of disruptive selection in $x$ (measured by $D_{x}=\sigma_{\alpha}^{-2}-\sigma_{K}^{-2}$ ) and by the strength of directional selection in $y$ (measured by $\left.G_{y}\right)$ ?

In line with the qualitative results already described above, we find that adaptive radiations are inhibited when directional selection in $y$ is too strong relative to disruptive selection in $x$ (figure 2). The corresponding critical relation between $D_{x}$ and $G_{y}$ roughly follows a linear relationship, $D_{x} \propto G_{y}$, in figure 2. Evolutionary community formation through adaptive radiations occurs for a wide range of selection strengths. Weaker directional selection in $y$ and stronger disruptive selection in $x$ both facilitate the build-up of larger communities (figure 2), while stronger directional selection in $y$ implies a more rapid turnover of species in the community.

Since the asexual individual-based model uses only the growth rate $b(x, y)-d(x, y)$ (Appendix B), different combinations of $b_{1}$ and $d_{1}$ sharing the same value of $b_{1}-d_{1}$ (e.g., $d_{1}=G_{y}$ and $b_{1}=0$ ) result in identical dynamics. More generally, recurrent radiations and extinctions are expected whenever $\left(D_{x}, G_{y}\right)$, i.e., the combination of the strength of disruptive selection in $x$ and of directional selection in $y$, stays within the gray region in figure 2. Birth and death rates can thus depend on time or on other variables not considered here, as long as $\left(D_{x}, G_{y}\right)$ moves within the gray region. For example, replac- 
ing $G_{y}(y-\bar{y})$ with $G_{y}\left(y-y_{0}\right)$, using a constant $y_{0}$ or time-dependent variable $y_{0}=a \cdot t$, can also induce recurrent radiations and extinctions.

\section{Robustness: sexual reproduction}

Adaptive radiations in sexual populations require reproductive isolation. While our model with sexual reproduction is ecologically equivalent to the model with asexual reproduction analyzed so far, it additionally allows for the emergence of prezygotic isolation through the divergence of male display and female preference traits.

Our results show that, as expected from the asexual model, the sexual population first directionally evolves to $x=0$. The disruptive frequency-dependent selection emerging at this evolutionary branching point then favors the pairwise divergence of male display trait $m$ and female preference trait $p$ (figure 3a), triggered by small linkage disequilibria among $x, m$, and $p$ (a similar effect was described by Dieckmann \& Doebeli 1999). Subsequently, the divergence in mating traits $m$ and $p$ restricts interbreeding between the two lineages and enables their divergence in ecological traits $x$ and $y$. Although disruptive selection weakens as interbreeding becomes rarer, divergence in $m$ and $p$ continues driven by genetic drift (Gavrilets 1997), leading to complete reproductive isolation (figure 3a). This process of adaptive radiation accompanied by reproductive isolation occurs repeatedly, and the divergence among lineages in terms of trait $y$ induces the extinction of posterior lineages (figure 3b). The depicted macroevolutionary dynamics of ecological traits in the sexual model are thus equivalent to those observed in the asexual model.

\section{Robustness: full two-dimensional model}

Macroevolutionary dynamics of repeated adaptive radiation and extinction also arise in the full twodimensional model, both for asexual populations (figure $4 \mathrm{a}$ ) and for sexual populations (figures $4 \mathrm{~b}$ and 4c). 
Robustness: mating systems

For the sexual model discussed so far we assumed that mating probabilities are determined by a male display trait and a female preference trait, and that the birth rates of males and females together match those in the asexual model. However, large variations exist among the mating systems of organisms. For example, mating probabilities might rather depend on ecologically neutral traits expressed by males and females (mating variant 1) or on the ecological traits $x$ and $y$ (mating variant 2). These variants are especially important for describing ecological settings in which prezygotic isolation is brought about by habitat choice. Moreover, intrinsic rates of reproduction might not differ among males, so that the effective reproduction rates of males just depend on how often they are chosen by females (mating variant 3). Further, the mating success, and thus the effective birth rate, of females preferring rare male display traits might be reduced, resulting in a cost of choosiness (mating variant 4).

We have checked the robustness of our results for sexual populations against these variations of the mating system. Implementation details are provided in Appendix D. Mating variants 1, 2, and 3 consistently result in recurrent adaptive radiations and extinctions. Mating variant 4 requires a sufficiently small cost of choosiness and sufficiently high mutation rates for the mating traits. A combination of mating variants 2 and 4 enables repeated radiations and extinctions also for larger costs of choosiness; see Appendix D.

\section{Robustness: spatial structure}

The requirements for recurrent adaptive radiations above are considerably relaxed when spatial structure is involved. We assumed that the carrying capacity's maximum gradually changes along a linear chain of patches connected by offspring migration. In each of these patches, birth and death events occur according to the simplified two-dimensional model as described above.

In such a parapatric setting, recurrent adaptive radiations and extinctions robustly occur under all four mating variants. In particular, for mating variant 4 they occur for larger costs of choosiness and/or 
for smaller mutation rates than in the corresponding non-spatial model, if the migration probability is sufficiently low; see Appendix E. This is just as expected, since the limited gene flow resulting from low migration rates favors the divergence of mating traits between patches. Migration of phenotypes from an anterior lineage into patches with posterior lineages thus promotes the latter's extinction without interbreeding.

We thus conclude that parapatric ecological settings further facilitate macroevolutionary processes of recurrent adaptive radiations and extinctions that, under more restrictive conditions, can already occur in sympatry.

\section{Robustness: other tests}

In the simplified two-dimensional model with sexual reproduction, repeated adaptive radiations and extinctions were also observed for increased abundances (e.g., for total population sizes of around 3000 individuals), for smaller mutation probabilities of mating traits and higher choosiness (e.g., for $\mu_{m}=\mu_{p}=10^{-4}$ and $\sigma_{m}=2.0$ ), and for smaller numbers of loci coding for each mating trait (e.g., for ten loci). Although $d_{1}=0$ was used for figures 3 and 4 , different combinations of $b_{1}$ and $d_{1}$ (including those with $b_{1}=0$ ) do not result in any qualitative change of the presented results. Other modifications, for example, replacing $b_{1} \cdot(y-\bar{y})$ with $b_{1} \cdot\left(y-y_{0}\right)$, using a constant $y_{0}$ or a time-dependent $y_{0}=a \cdot t$, also induce recurrent radiation and extinction, as long as $D_{x}$ is sufficiently larger than $G_{y}$ and $a$ is small. Furthermore, we found that the explicit implementation of growth and ageing of individuals did not qualitatively change the observed macroevolutionary dynamics.

\section{Discussion}

\section{Evolutionary branching in multivariate traits}

The theory of adaptive dynamics has provided a general conceptual framework for understanding and analyzing the ecological conditions underlying processes of evolutionary diversification driven by eco- 
logical interactions (see Dieckmann et al. 2004 for an overview). However, most of the corresponding models (see Introduction) are based on one-dimensional quantitative traits. In that case, adaptive radiations are expected to occur at evolutionary branching points, i.e., at points in trait space where directional selection ceases and frequency-dependent disruptive selection remains (Metz et al. 1992, 1996; Geritz et al. 1997, 1998).

Under the assumption of infinitesimal mutation probabilities and mutational step sizes, the necessary conditions for evolutionary branching can be carried over from one-dimensional to higher-dimensional trait spaces: in particular, evolutionary branching will occur only where directional selection ceases in all directions. This would imply, however, that evolutionary branching in a focal trait cannot happen unless directional selection in all jointly evolving traits vanishes completely. Since all organisms possess more than a single evolving trait, that would make evolutionary branching all but impossible.

In reality, of course, mutation probabilities and mutational step sizes may be small but are always finite. The results presented in this study illustrate that, under these natural circumstances, evolutionary branching in multivariate quantitative traits may occur much more generally, whenever frequencydependent disruptive selection in some trait directions is sufficiently strong compared with directional selection in the remaining trait directions. For understanding the occurrence of evolutionary branching in higher-dimensional trait spaces, traits that are under only weak directional selection can thus just as well be left out. This conclusion, however, does not extend to the eventual macroevolutionary patterns: when time scales are considered that are sufficiently long for such weakly selected traits to evolve significantly, they qualitatively affect the expected evolutionary outcomes. In particular, as shown above, recurrent adaptive radiations and extinctions can thus be induced.

It is interesting to note that the boundary in figure 2 implies a linear relationship between the strength of disruptive selection in $x, D_{x}$, and the strength of directional selection in $y, G_{y}$. Indeed, it can be shown analytically that, for an asexual populations, the likelihood of the first evolutionary 
branching is determined by $\left(\sigma_{\mu x}^{2} D_{x}\right) /\left(\sigma_{\mu y} G_{y}\right)$, irrespective of the types of ecological interaction that influence birth and death rates (Ito \& Dieckmann, unpublished).

\section{Generality of macroevolutionary mechanism}

The results presented here show how frequency-dependent disruptive selection in one trait, combined with weak directional selection in another trait, naturally and robustly lead to macroevolutionary patterns of recurrent adaptive radiations and extinctions.

Such a combination of selection pressures must be expected to be common in nature. This is because frequency-dependent disruptive selection in univariate traits is predicted to occur under a wide variety of circumstances, in very different quantitative characters, and for many types of ecological interactions (see Doebeli \& Dieckmann 2000 and references cited in the Introduction). Owing to the high dimensionality of real trait spaces and the ubiquity of externally driven changes in the ecological environments of organisms, some residual weak directional selection in at least one other trait direction can always be taken for granted. The requirements for inducing recurrent adaptive radiations and extinctions thus appear to be readily met in nature.

The evolutionary mechanisms underlying such incessant macroevolution are not difficult to understand, and have been explained in detail above: while lineages best adapted to the directional selection pressures inevitably emerge through spontaneous symmetry breaking, they subsequently experience a positive feedback in fitness that drives other lineages to extinction, before the extinct lineages are replaced through adaptive radiations enabled by frequency-dependent disruptive selection.

Macroevolutionary patterns broadly similar to those analyzed here have occurred repeatedly in evolutionary history. Examples include the radiation of angiosperms followed by the decline of gymnosperms (Wing and Boucher 1998) and the diversification of eutherian mammals replacing metatherian mammals (Lillegraven 1979). 
Experimental tests of the macroevolutionary mechanism proposed here might be feasible in microbial organisms, in which adaptive radiations due to frequency-dependent selection have already been demonstrated (MacLean 2005). Including a second kind of trait and selection pressure - for example, by considering a character $y$ for temperature adaptation, in addition to a character $x$ for resource competition - could immediately enable testing our predictions in real asexual populations.

\section{Taxon cycles}

The results presented here have some bearing on the discussion of taxon cycles (Wilson 1961), which are based on likening the aging of species to that of individuals. In accordance with this perspective, we can indeed show that in our model the fitness component of lineages in the direction of directional selection monotonically decreases with a lineage's age (figure 5). This monotonicity underlies the 'developmental cycle' of lineages and is caused by the positive feedback between a lineage's fitness and its evolving position along the trait dimension subject to directional selection.

Cycles of evolutionary branching and extinction akin to taxon cycles had already been found in earlier theoretical studies (e.g., Taper \& Case 1992; Kisdi et al. 2001). Such alternative models of taxon cycles, however, consistently had to rely on asymmetric ecological interactions. Here we have shown that recurrent adaptive radiations and extinctions can occur even when all considered ecological interactions are strictly symmetric. In our model, asymmetry in the competition between lineages is of evolutionary rather than ecological origin and stems from a positive feedback mechanism that causes evolutionary divergence in a trait under weak directional selection.

The models presented here can also be employed to examine the lifetime of lineages (figure 5). Further work in this direction will help to complement other theoretical studies predicting lifetime distributions, including those based on self-organized criticality (Bak 1997; Pigolotti et al. 2005). We find that large values of $D_{x} / G_{y}$ and $G_{y}$ favor frequent radiations and extinctions, respectively. Such combinations are thus expected to shorten the lifetimes of lineages. 


\section{Summary}

In this study we have demonstrated how the straightforward extension of well-established univariate models of frequency-dependent disruptive selection, through the inclusion of a second trait under weak directional selection, induces recurrent adaptive radiations and extinctions, both in asexual and in sexual populations. That these macroevolutionary patterns can be understood in terms of general geometric features of bivariate frequency-dependent fitness landscapes explains their robustness. This means that the processes of recurrent adaptive radiations and extinctions reported here must be expected to occur even when the specific ecological underpinnings responsible for disruptive and directional selection pressures are altered. We thus hope that - by offering an abstract and general framework, with minimal ingredients - our model may serve as a stepping stone for understanding macroevolutionary phenomena of wide-ranging relevance.

\section{Acknowledgements}

The authors thank Michael Doebeli, Géza Meszéna, Hans Metz, Masakazu Shimada, and two anonymous reviewers and editors for valuable comments on earlier versions of this manuscript. H.I. also thanks David Munro for inventing a tool for numerical analysis and visualization with great performance and flexibility, named Yorick, and distributing it for free. All figures in this study were produced with Yorick. H.I. acknowledges support in the form of a Research Fellowship for Young Scientists by the Japan Society for the Promotion of Science (JSPS), and by IIASA's Adaptive Dynamics Network, facilitating his participation in the Young Scientists Summer Program (YSSP) of the International Institute for Applied Systems Analysis (IIASA). U.D. gratefully acknowledges financial support by the Austrian Science Fund (FWF); by the Austrian Federal Ministry of Education, Science, and Cultural Affairs (bm:bwk); by the Vienna Science and Technology Fund (WWTF); and by the Marie Curie Research Training Network ModLife (Modern Life-History Theory and its Application to the 
Management of Natural Resources) funded through the Human Potential Programme of the European Commission.

\section{Appendix A: Scope of simplified two-dimensional model}

Models such as (3) are built on the simplifying assumption that the demographic effects of a population's distribution of character values can be captured through a character's average value. Models of this kind have been used expensively for studying the evolutionary ecology of frequency-dependent interactions (e.g., Abrams et al. 1993) and are known to approximate more general models when the considered character's variance is sufficiently small (Iwasa et al. 1991; Taper and Case 1992).

Applying this rationale to the character $y$ in our two-dimensional model, we can express the frequency dependence in $y$ of, for example, the population's per capita birth rate as $b(x, y, \bar{y})$. Here the population's mean in the character $y$ is $\bar{y}=\sum_{j} y_{j} n_{j} / \sum_{j} n_{j}$, and its variance in $y$, $\sigma_{y}^{2}=\sum_{j} n_{j}\left(y_{j}-\bar{y}\right)^{2} / \sum_{j} n_{j}$, is assumed to be sufficiently small. The first-order Taylor expansion of $b(x, y, \bar{y})$ in $y$ around $\bar{y}$,

$$
b(x, y, \bar{y}) \approx b_{0}(x, \bar{y})+b_{1}(x, \bar{y}) \cdot(y-\bar{y})
$$

will then be a good approximation, since differences $y-\bar{y}$ will be small.

To recover (3a), we thus need to assume that (a) the variance in the character $y$ is not too large, so that $\sigma_{y}^{2}$ is small (relative to the scale of potential nonlinearities in the dependence of $b$ on $y$ ), (b) the baseline birth rate is determined by the character $x$, so that $b_{0}(x, \bar{y})=b(x)$, and (c) the sensitivity of $b$ to variation of $y$ around $\bar{y}$ is constant, so that $b_{1}(x, \bar{y})=b_{1}$. The requirements for the per capita death rate $d$ to be given by (3b) are analogous. The resultant set of assumptions thus delimits the conditions under which the simplified model (3) is applicable. 


\section{Appendix B: Asexual model}

To enhance the computational tractability of long-term asexual evolution driven by mutation and selection, we followed the usual assumption that evolutionary dynamics occur on a slower time scale than population dynamics. In the corresponding approximation (monomorphic stochastic model; Dieckmann 1994; Dieckmann et al. 1995; Dieckmann \& Law 1996), mutations are rare, so that evolution can be described as a directed random walk. In each step of this random walk, a mutant phenotype successfully replaces its ancestor. In the absence of evolutionary branching, invasion of a mutant phenotype generically implies replacement of the former resident phenotype (Geritz et al. 2002). Here we have extended this model to include evolutionary branching, resulting in what we call the oligomorphic stochastic model.

The algorithm of the asexual model is listed below:

0 . Initialize the phenotypes $s=\left(s_{1}, \ldots, s_{N}\right)$ of all $N$ resident lineages $k=1, \ldots, N$ at time $t=0$ (an initial value of $N=1$ is used throughout this study, corresponding to an initially monomorphic community). Calculate equilibrium population sizes $\hat{n}=\left(\hat{n}_{1}, \ldots, \hat{n}_{N}\right)$ at which $b\left(s_{k}\right)=d\left(s_{k}\right)$ for all $k=1, \ldots, N$. Define the extinction threshold $\varepsilon$.

1. Calculate the rate $w_{k}=\mu \hat{n}_{k} b\left(s_{k}\right)$ for the emergence of a mutant from phenotype $s_{k}$, as well as the total rate $w=\sum_{k=1}^{N} w_{k}$, where $\mu$ is the mutation rate.

2. Choose lineage $i$ with probability $w_{i} / w$.

3. Choose a new phenotype $s_{i}^{\prime}$ according to the mutation probability density $\Omega\left(s_{i}^{\prime}-s_{i}\right)$. Update time $t$ by adding $\Delta t=-\frac{1}{w} \ln \rho$, where $0<\rho \leq 1$ is a uniformly distributed random number. Calculate the invasion fitness $f\left(s_{i}^{\prime}\right)=b\left(s_{i}^{\prime}\right)-d\left(s_{i}^{\prime}\right)$ of the new phenotype at $\hat{n}$.

4. Choose a uniformly distributed random number $0<\rho \leq 1$. If $\rho \geq f\left(s_{i}^{\prime}\right) / b\left(s_{i}^{\prime}\right)$, return to Step 2. Else, calculate equilibrium population sizes $\hat{n}^{\prime}=\left(\hat{n}_{1}^{\prime}, \ldots, \hat{n}_{i}^{\prime}, \ldots, \hat{n}_{N}^{\prime}\right)$ for $s^{\prime}=\left(s_{1}, \ldots, s_{i}^{\prime}, \ldots, s_{N}\right)$. 
5. If $\hat{n}_{k}^{\prime}>\varepsilon$ for all $k=1, \ldots, N$ and $f\left(s_{i}\right)<0$ at $\hat{n}^{\prime}$, replace $s_{i}$ with $s_{i}^{\prime}$ and $\hat{n}$ with $\hat{n}^{\prime}$, and continue with Step 6. Else, increase $N$ by 1 and set $s_{N}=s_{i}^{\prime}$. Calculate equilibrium population sizes from population dynamics with initial population sizes $n_{k}=\hat{n}_{k}$ for $k=1, \ldots, N-1$ and $n_{N}=\varepsilon$. In the course of these population dynamics, delete phenotypes $s_{k}$ with $\hat{n}_{k}<\varepsilon$, and decrease $N$ accordingly.

6. Continue with Step 1.

\section{Appendix C: Sexual model}

Sexual reproduction is implemented on an individual basis. The variables $M_{i}$ and $F_{i}=1-M_{i}$ are used to indicate the sex of individual $i: M_{i}=1$ if $i$ is male, and $F_{i}=1$ if $i$ is female.

The algorithm of the sexual model is listed below:

0 . Initialize the genotypes and sexes of all $N$ individuals $k=1, \ldots, N$ at time $t=0$. Calculate the birth rates $b\left(s_{k}\right)$ and death rates $d\left(s_{k}\right)$ of all individuals $k=1, \ldots, N$.

1. Construct the sums $w_{d}=\sum_{k=1}^{N} d\left(s_{k}\right), w_{b}=\sum_{k=1}^{N} 2 b\left(s_{k}\right) F_{k}$, and $w=w_{b}+w_{d}$.

2. Choose a death or birth event with probabilities $w_{d} / w$ and $w_{b} / w$, respectively. If a death event is chosen, continue with Step 3; else, continue with Step 4.

3. Choose individual $i$ with probability $d\left(s_{i}\right) / w_{d}$ and remove it from the population. Decrease total population size $N$ by 1 . Continue with Step 5 .

4. Choose female individual $i$ with probability $2 b\left(s_{i}\right) / w_{b}$. Construct the sum $w_{M}=\sum_{k=1}^{N} b\left(s_{k}\right) P_{i k} M_{k}$. Choose male individual $j$ with probability $b\left(s_{j}\right) P_{i j} / w_{M}$. Assemble two haploid gametes through recombination from individuals $i$ and $j$. Combine these gametes into the genotype of a diploid offspring, and toggle alleles at each locus according to trait-specific mutation probabilities $\mu_{x}, \mu_{y}$, 
$\mu_{m}$, and $\mu_{p}$. Add a new individual with this genotype to the population, and assign its sex at random. Increase total population size $N$ by 1 .

5. Update time $t$ by adding $\Delta t=-\frac{1}{w} \ln \rho$, where $0<\rho \leq 1$ is a uniformly distributed random number. Continue with Step 1.

\section{Appendix D: Mating systems}

We checked the robustness of our results against the following variations in the mating system:

Mating variant 1: Mating depends on ecologically neutral traits $q=\left(q_{1}, \ldots, q_{L_{q}}\right)$ expressed by males and females. Equation (4c) is replaced with $d_{i j}^{2}=\sum_{k=1}^{L_{q}}\left(q_{i k}-q_{j k}\right)^{2}$.

Mating variant 2: Mating depends on both ecological traits. Equation (4c) is replaced with $d_{i j}^{2}=\beta^{2}\left[\left(x_{i}-x_{j}\right)^{2}+\left(y_{i}-y_{j}\right)^{2}\right]$ (where $\beta=10$ compensates for the difference in mutation probabilities of ecological traits and mating traits).

Mating variant 3: Intrinsic rates of reproduction do not differ among males. In Step 4 of Appendix C, a male individual $j$ is chosen with probability $P_{i j} / w_{M}$, where $w_{M}=\sum_{k=1}^{N} P_{i k} M_{k}$.

Mating variant 4: Following Doebeli \& Dieckmann (2003), a cost of choosiness is introduced. In Steps 1 and 4 of Appendix $C$, the intrinsic birth rate $2 b\left(s_{i}\right)$ of female individuals is replaced with the effective birth rate $2 b\left(s_{i}\right) /\left(1+B_{c} / B_{i}\right)$, where $B_{i}=\sum_{j=1}^{N} b\left(s_{j}\right) P_{i j} M_{j}$ is the number of suitable mating partners. The parameter $B_{c}$ measures the value of $B_{i}$ at which the effective birth rate drops to half the intrinsic birth rate.

As described in the main text, recurrent radiation and extinction readily arises under mating variants 1 and 3 , as well as under variant 2 if assortative mating is sufficiently strong (e.g., $\sigma_{m}=5$ ). For variant 
4 alone, small costs of choosiness are required (e.g., $B_{c}=0.2$ ). A combination of mating variants 2 and 4 enables recurrent radiation and extinction at larger costs of choosiness (e.g., $B_{c}=5$ for $\left.\sigma_{m}=5\right)$.

\section{Appendix E: Spatial structure}

We introduced spatial structure into the simplified two-dimensional model by considering a linear chain of $N_{z}$ patches labeled $z=1, \ldots, N_{z}$. The carrying capacity distribution in the $z$ th patch is given by

$$
K(x, z)=K_{0} \exp \left(-\frac{1}{2}\left(x-z d_{z}\right)^{2} / \sigma_{K}^{2}\right),
$$

where $d_{\mathrm{z}}$ measures the difference between adjacent patches in the positions of the maximum carrying capacity. The abundance of extant phenotypes $j=1, . ., N$ in patch $z$ is denoted by $n_{j z}$. The per capita birth and death rates of individuals with characters $(x, y)$ in the $z$ th patch are given by

$$
\begin{gathered}
b(x, y, z)=r\left[1+G_{y}\left(y-\bar{y}_{z}\right)\right], \\
d(x, y, z)=r \sum_{j} \alpha\left(x-x_{j}\right) n_{j z} / K(x, z),
\end{gathered}
$$

where $\bar{y}_{z}$ is the average value of $y$ in the zth patch. Individuals mate within patches. Offspring migrate with probability $\xi$ between adjacent patches.

Assuming sexual reproduction and mating variant 4, we could show that this spatial structure enables recurrent adaptive radiations even for large costs of choosiness and/or small mutation rates, if only the migration probability $\xi$ is sufficiently low. For example, radiations occur for $B_{c}=5$, $\mu_{m}=\mu_{p}=5 \times 10^{-4}$, and $\xi=5 \times 10^{-3}$, as well as for $B_{c}=5, \mu_{m}=\mu_{p}=5 \times 10^{-5}$, and $\xi=5 \times 10^{-4}$. Other model parameters: $N_{z}=3, d_{z}=0.15, K_{0}=500, r=1, \sigma_{K}=\sigma_{\alpha}=0.14, G_{y}=7.5, \sigma_{m}=5, L_{x}=40$, $L_{y}=10, L_{m}=L_{p}=15, \mu_{x}=10^{-5}, \mu_{y}=5 \times 10^{-6}, C_{x}=4, C_{y}=1$, and $C_{m}=C_{p}=5$. 


\section{Literature Cited}

Abrams, P. A., Matsuda, H. \& Harada, Y. 1993 Evolutionarily unstable fitness maxima and stable fitness minima of continuous traits. Evol. Ecol. 7, 465-487.

Ackermann, M. \& Doebeli, M. 2004 Evolution of niche width and adaptive diversification. Evolution 58, 2599-2612.

Bak, P. 1997 How nature works: The science of self-organized criticality. Oxford University Press.

Bolnick, D. I. 2004 Waiting for sympatric speciation. Evolution 58, 895-899.

Bonsall, M. B., Jansen, V. A. A. \& Hassell, M. P. 2004 Life history trade-offs assemble ecological guilds. Science 306, 111-114.

Boots, M. \& Haraguchi, Y. 1999 The evolution of costly resistance in host-parasite systems. Am. Nat. 153, 359-370.

Bowers, R. G., White, A., Boots, M., Geritz, S. A. H. \& Kisdi, E. 2003 Evolutionary branching/speciation: contrasting results from systems with explicit or emergent carrying capacities. Evol. Ecol. Res. 5, 883-891.

Brown, J. S. \& Pavlovic, N. B. 1992 Evolution in heterogeneous environments: Effects of migration on habitat specialization. Evol. Ecol. 6, 360-382.

Brown, W. L. \& Wilson, E. O. 1956 Character displacement. Syst. Zool. 5, 49-64.

Bush, G. L. 1975 Modes of animal speciation. Ann. Rev. Ecol. Syst. 6, 339-364.

Case, T. J. 1981 Niche packing and coevolution in competition communities. Proc. Natl. Acad. Sci. USA 78, 5021-5025.

Cheptou, P. O. \& Mathias, A. 2001 Can varying inbreeding depression select for intermediary selfing rate? Am. Nat. 157, 361-373

Chow, S. S., Wilke, C. O., Ofria, C., Lenski, R. E. \& Adami, C. 2004 Adaptive radiation from resource competition in digital organisms. Science 305, 84-86. 
Christiansen, F. B. 1991 On conditions for evolutionary stability for a continuously varying character. Am. Nat. 138, 37-50.

Christiansen, F. B. \& Fenchel, T. M. 1977 Theories of populations in biological communities. Berlin, Germany: Springer-Verlag.

Christiansen, F. B. \& Loeschcke, V. 1980 Evolution and intraspecific exploitative competition. I. One locus theory for small additive gene effects. Theor. Popul. Biol. 18, 297-313.

Claessen, D. \& Dieckmann, U. 2002 Ontogenetic niche shifts and evolutionary branching in sizestructured populations. Evol. Ecol. Res. 4, 189-217.

Cohen, Y., Vincent, T. L. \& Brown, J. S. 1999 A G-function approach to fitness minima, fitness maxima, evolutionarily stable strategies and adaptive landscapes. Evol. Ecol. Res. 1, 923-942.

Day, T. 2000 Competition and the effect of spatial resource heterogeneity on evolutionary diversification. Am. Nat. 155, 790-803.

Day, T. \& Young, K. A. 2004 Competitive and facilitative evolutionary diversification. Bioscience 54, 101-109.

Dayan, T. \& Simberloff, D. 2005 Ecological and community-wide character displacement: the next generation. Ecol. Lett. 8, 875-894.

De Jong, T. \& Geritz, S. A. H. 2001 The role of geitonogamy in the gradual evolution towards dioecy in cosexual plants. Selection 2, 133-146.

Dercole, F. 2003 Remarks on branching-extinction evolutionary cycles. J. Math. Biol. 47, 569-580.

Dieckmann, U. 1994 Coevolutionary dynamics of stochastic replicator systems. Master's thesis, University of Aachen, Germany, and Central Library of the Research Center Juelich, Germany. Available online at http://www.iiasa.ac.at/ dieckman.

Dieckmann, U. \& Doebeli, M. 1999 On the origin of species by sympatric speciation. Nature 400, 354357. 
Dieckmann, U., Marrow, P. \& Law, R. 1995 Evolutionary cycling in predator-prey interactions: Population dynamics and the Red Queen. J. Theor. Biol. 176, 91-102.

Dieckmann, U., Metz, J. A. J., Doebeli, M. \& Tautz, D., editors 2004 Adaptive speciation. Cambridge University Press.

Dieckmann, U. \& Doebeli, M. 2004 Adaptive dynamics of speciation: Sexual populations. In Adaptive speciation (eds. Dieckmann, U., Metz, J. A. J., Doebeli, M. \& Tautz, D.), pp 76-111. Cambridge University Press.

Doebeli, M. \& Dieckmann, U. 2003 Speciation along environmental gradients. Nature 421, 259-264.

Doebeli, M. \& Dieckmann, U. 2005 Adaptive dynamics as a mathematical tool for studying the ecology of speciation processes. J. Evol. Biol. 18, 1194-1200.

Doebeli, M., Dieckmann, U., Metz, J. A. J. \& Tautz, D. 2005 What we have also learned: Adaptive speciation is theoretically plausible. Evolution 59, 691-695.

Drossel, B. \& McKane, A. 2000 Competitive speciation in quantitative genetic models. J. Theor. Biol. 204, 467-478.

Doebeli, M. \& Dieckmann, U. 2000 Evolutionary branching and sympatric speciation caused by different types of ecological interactions. Am. Nat. 156, S77-S101.

Doebeli, M. 1996a A quantitative genetic model for sympatric speciation. J. Evol. Biol. 9, 893-909.

Doebeli, M. 1996b An explicit genetic model for ecological character displacement. Ecology 77, 510520.

Doebeli, M. 2002 A model for the evolutionary dynamics of cross-feeding polymorphisms in microorganisms. Popul. Ecol. 44, 59-70.

Doebeli, M. \& Ruxton, G. D. 1997 Evolution of dispersal rates in metapopulation models: Branching and cyclic dynamics in phenotype space. Evolution 51, 1730-1741.

Doebeli, M., Blok, H. J., Leimar, O. \& Dieckmann, U. 2007 Multimodal pattern formation in phenotype distributions of sexual populations. Proc. R. Soc. Lond. B 274, 347-357 
Doebeli, M., Hauert, C. \& Killingback, T. 2004 The evolutionary origin of cooperators and defectors. Science 306, 859-862.

Egas, M., Dieckmann, U. \& Sabelis, M. W. 2004 Evolution restricts the coexistence of specialists and generalists: The role of trade-off structure. Am. Nat. 163, 518-531.

Egas M., Sabelis, M. W. \& Dieckmann, U. 2005 Evolution of specialization and ecological character displacement of herbivores along a gradient of plant quality. Evolution 59, 507-520.

Ernande, B. \& Dieckmann, U. 2004 The evolution of phenotypic plasticity in spatially structured environments: Implications of intraspecific competition, plasticity costs and environmental characteristics. J. Evol. Biol. 17, 613-628.

Feder, J. L., Roethele, J. B., Wlazlo, B. \& Berlocher, S. H. 1997 Selective maintenance of allozyme differences among sympatric host races of the apple maggot fly. Proc. Natl. Acad. Sci. USA 94, 11417-11421.

Felsenstein, J. 1981 Scepticism towards Santa Rosalia, or why are there so few kinds of animals? Evolution 35, 124-138.

Ferdy, J. B., Despres, L. \& Godelle, B. 2002 Evolution of mutualism between globeflowers and their pollinating flies. J. Theor. Biol. 217, 219-234.

Ferrière, R., Bronstein, J. L., Rinaldi, S., Law, R. \& Gauduchon, M. 2002 Cheating and the evolutionary stability of mutualisms. Proc. $R$. Soc. Lond. B 269, 773-780.

Gavrilets, S. 1997 Evolution and speciation on holey adaptive landscapes. Trends Ecol. Evol. 12, 307312.

Gavrilets, S. 2005 “Adaptive speciation”: it is not that simple. Evolution 59, 696-699.

Geritz, S. A. H., Gyllenberg, M., Jacobs, F. J. A. \& Parvinen, K. 2002. Invasion dynamics and attractor inheritance. J. Math. Biol. 44, 548-560.

Geritz, S. A. H., Kisdi, É., Meszéna, G. \& Metz, J. A. J. 1998 Evolutionarily singular strategies and the adaptive growth and branching of the evolutionary tree. Evol. Ecol. 12, 35-57. 
Geritz, S. A. H., Metz, J. A. J., Kisdi, É. \& Meszéna, G. 1997 The dynamics of adaptation and evolutionary branching. Phys. Rev. Lett. 78, 2024-2027.

Geritz, S. A. H., Van der Meijden, E. \& Metz, J. A. J. 1999 Evolutionary dynamics of seed size and seedling competitive ability. Theor. Popul. Biol. 55, 324-343.

Gillespie, D. T. 1976 A general method for numerically simulating the stochastic time evolution of coupled chemical reactions. J. Comp. Phys. 22, 403-434.

Gould, S. J. \& Eldredge, N. 1977. Punctuated equilibria: the tempo and mode of evolution reconsidered. Paleobiology 3, 115-151.

Grant, P. R. 1998 Evolution on islands. Oxford University Press, Oxford.

Gudelj, I., van den Bosch, F. \& Gilligan, C. A. 2004 Transmission rates and adaptive evolution of pathogens in sympatric heterogeneous plant populations Proc. R. Soc. Lond. B 271, 2187-2194.

Iwasa, Y., Pomiankowski, A. \& Nee, S. 1991 The evolution of costly mate preferences. II. The 'handicap' principle. Evolution 45, 1431-1442.

Jansen, V. A. A. \& Mulder, G. S. E. E. 1999 Evolving biodiversity. Ecol. Lett. 2, 379-386.

Johst, K., Doebeli, M. \& Brandl, R. 1999 Evolution of complex dynamics in spatially structured populations Proc. R. Soc. Lond. B 266, 1147-1154.

Kisdi, É. 2001 Long-term adaptive diversity in Levene-type models. Evol. Ecol. Res. 3, 721-727.

Kisdi, É. 1999 Evolutionary branching under asymmetric competition. J. Theor. Biol. 197, 149-162.

Kisdi, É. \& Geritz, S. A. H. 1999 Adaptive dynamics in allele space: Evolution of genetic polymorphism by small mutations in a heterogeneous environment. Evolution 53, 993-1008.

Kisdi, É. \& Geritz, S. A. H. 2001 Evolutionary disarmament in interspecific competition. Proc. R. Soc. Lond. B 268, 2589-2594.

Kisdi, É., Jacobs, F. J. A. \& Geritz, S. A. H. 2001 Red Queen evolution by cycles of evolutionary branching and extinction. Selection 2, 161-176. 
Koella, J. C. \& Doebeli, M. 1999 Population dynamics and the evolution of virulence in epidemiological models with discrete host generations. J. Theor. Biol. 198, 461-475.

Kondrashov, A. S. \& Kondrashov, F. A. 1999 Interactions among quantitative traits in the course of sympatric speciation. Nature 400, 351-354.

Law, R., Bronstein, J. L. \& Ferrière, R. 2001 On mutualists and exploiters: Plant-insect coevolution in pollinating seed-parasite systems. J. Theor. Biol. 212, 373-389.

Leimar, O. 2005 The evolution of phenotypic polymorphism: Randomized strategies versus evolutionary branching. Am. Nat. 165, 669-681.

Lillegraven, J. A. 1979 Reproduction in Mesozoic mammals. In Mesozoic mammals: The first twothirds of mammalian history (ed. J.A. Lillegraven, Z. Kielan-Jaworowska \& W.A. Clemens), pp 259-276. Berkeley: University of California Press.

Loeuille, N. \& Loreau, M. 2005 Evolutionary emergence of size-structured food webs. Proc. Natl. Acad. Sci. USA 102, 5761-5766.

MacArthur, R. H. 1972 Geographical ecology. New York: Harper and Row.

MacLean, R. C. 2005 Adaptive radiation in microbial microcosms. J. Evol. Biol. 18, 1376-1386.

Maire N., Ackermann, M. \& Doebeli, M. 2001 Evolutionary branching and the evolution of anisogamy. Selection 2, 119-132.

Matessi, C., Gimelfarb, A. \& Gavrilets, S. 2001 Long term buildup of reproductive isolation promoted by disruptive selection: how far does it go? Selection 2, 41-64.

Mathias, A. \& Kisdi, É. 2002 Adaptive diversification of germination strategies. Proc. R. Soc. Lond. B 269, 151-156.

Mathias, A., Kisdi, É. \& Olivieri, I. 2001 Divergent evolution of dispersal in a heterogeneous landscape. Evolution 55, 246-259.

Maynard Smith, J. 1966 Sympatric speciation. Am. Nat. 100, 637-650. 
McKinney, M. L. \& Drake, J. A., editors 1998 Biodiversity dynamics: turnover of populations, taxa, and communities. Columbia University Press.

Meszéna, G., Czibula, I. \& Geritz, S. A. H. 1997 Adaptive dynamics in a 2-patch environment: A toy model for allopatric and parapatric speciation. J. Biol. Syst. 5, 265-284.

Meszéna, G. \& Szathmáry, E. 2001 Adaptive dynamics of parabolic replicators. Selection 2, 147-160.

Metz, J. A. J., Geritz, S. A. H., Meszéna, Jacobs, F. J. A. \& van Heerwaarden, J. S. 1996 Adaptive dynamics: A geometrical study of the consequences of nearly faithful reproduction. In Stochastic and spatial structures of dynamical systems (ed. S. J. van Strien \& S. M. Verduyn Lunel), pp. 183-231. North Holland: Elsevier.

Metz, J. A. J., Nisbet, R. M. \& Geritz, S. 1992 How should we define 'fitness' for general ecological scenarios? Trends Ecol. Evol. 7, 198-202.

Morris, S. C. 1998 The evolution of diversity in ancient ecosystems: a review. Phil. Trans. R. Soc. Lond. B 353, 327-345.

Orr, M. R. \& Smith, T. B. 1998 Ecology and speciation. Trends Ecol. Evol. 13, 502-506.

Parvinen, K. 1999 Evolution of migration in a metapopulation. Bull. Math. Biol. 61, 531-550.

Parvinen, K. \& Egas, M. 2004 Dispersal and the evolution of specialisation in a two-habitat type metapopulation. Theor. Popul. Biol. 66, 233-248.

Pigolotti, S., Flammini, A., Marsili, M. \& Maritan, A. 2005 Species lifetime distribution for simple models of ecologies. Proc. Natl. Acad. Sci. USA 102, 15747-15751.

Regoes, R. R., Nowak, M. A. \& Bonhoeffer, S. 2000 Evolution of virulence in a heterogeneous host population. Evolution 54, 64-71.

Reuter, M., Helms, K. R., Lehmann, L. \& Keller, L. 2004 Effects of brood manipulation costs on optimal sex allocation in social hymenoptera. Am. Nat. 164, E73-E82.

Rosenzweig, M. L. 1978 Competitive speciation. Biol. J. Linn. Soc. 10, 275-289. 
Roughgarden, J. 1974 Species packing and the competition function with illustrations from coral reef fish. Theor. Popul. Biol. 5, 163-186.

Roughgarden, J. 1976 Resource partitioning among competing species: A coevolutionary approach. Theor. Popul. Biol. 9, 388-424.

Schluter, D. 1994 Experimental evidence that competition promotes divergence in adaptive radiation. Science 266, 798-801.

Schluter, D. 2000 The ecology of adaptive radiations. Oxford University Press, Oxford.

Schreiber, S. J. \& Tobiason, G. A. 2003 The evolution of resource use. J. Math. Biol. 47, 56-78.

Seger, J. 1985 Intraspecific resource competition as a cause of sympatric speciation. In Evolution: Essays in honour of John Maynard Smith, eds. Greenwood, P. J., Harvey, P.H. \& Slatkin, M., pp. 4353. Cambridge University Press.

Slatkin, M. 1980 Ecological character displacement. Ecology 61, 163-177.

Taper, M. L. \& Case, T. J. 1985 Quantitative genetic models for the coevolution of character displacement. Ecology 66, 355-371.

Taper, M. L. \& Case, T. J. 1992 Models of character displacement and the theoretical robustness of taxon cycles. Evolution 46, 317-333.

Troost, T. A., Kooi, B. W. \& Kooijman, S. A. L. M. 2005 Ecological specialization of mixotrophic plankton in a mixed water column. Am. Nat. 166, E45-E61.

Udovic, D. 1980 Frequency-dependent selection, disruptive selection, and the evolution of reproductive isolation. Am. Nat. 116, 621-641.

Van der Laan, J. D. \& Hogeweg, P. 1995 Predator-prey coevolution: Interactions across different timescales. Proc. R. Soc. Lond. B 259, 35-42.

Van Dooren, T. J. M. \& Leimar, O. 2003 The evolution of environmental and genetic sex determination in fluctuating environments. Evolution 57, 2667-2677. 
Van Doorn, G. S., Dieckmann, U. \& Weissing, F. J. 2004 Sympatric speciation by sexual selection: A critical re-evaluation. Am. Nat. 163, 709-725.

Van Doorn, G. S., Luttikhuizen, P. C. \& Weissing, F. J. 2001 Sexual selection at the protein level drives the extraordinary divergence of sex-related genes during sympatric speciation. Proc. R. Soc. Lond. B 268, 2155-2161.

Van Valen, L. 1973 A New Evolutionary Law. Evol. Theory 1, 1-30.

Vincent, T. L., Cohen, Y. \& Brown, J. S. 1993 Evolution via strategy dynamics. Theor. Popul. Biol. 44, 149-176.

Vukics, A., Asboth, J. \& Meszéna, G. 2003 Speciation in multidimensional evolutionary space. Phys. Rev. E 68, 041903.

Wilson, E. O. 1961 The nature of the taxon cycle in the Melanesian ant fauna. Am. Nat. 95, 169-193.

Wing, S. L. \& Boucher, L. D. 1998 Ecological aspects of the cretaceous flowering plant radiation. Ann. Rev. Earth Planet. Sci. 26, 379-421. 


\section{Figure caption}

Figure 1: Evolutionary dynamics in the simplified two-dimensional asexual model. Upper panels (A) to $(\mathrm{C})$ show the evolutionary dynamics for a medium width of the resource distribution in trait $x$ $\left(\sigma_{K}=0.2\right.$, compared with $\left.\sigma_{\alpha}=0.15\right)$. (A) No selection in trait $y, G_{y}=0$. (B), (C) Weak directional selection in trait $y, G_{y}=0.5$. Lower panels (D) to (E) show the evolutionary dynamics for a twice larger width of the resource distribution in trait $x\left(\sigma_{K}=0.2\right.$, compared with $\left.\sigma_{\alpha}=0.07\right)$. (D) No selection in trait $y, G_{y}=0$. (E), (F) Weak directional selection in trait $y, G_{y}=0.5$. In panels (A), (B), (D), and (E), the widths of lineages indicate their population size with linear scaling, while the colors of lineages (green to blue to red) indicate their trait value $y$ compared with the community's current average $\bar{y}$. In (C) and (F), the horizontal axis shows $G_{y}(y-\bar{y})$, i.e., the fitness component of lineages that derives from their trait $y$. Other parameters: $K_{0}=100,000, r=1, \sigma_{\mu x}=\sigma_{\mu y}=5 \times 10^{-3}, \mu=10^{-5}$, and $\varepsilon=1$.

Figure 2: Number of coexisting lineages in the simplified two-dimensional asexual model. The number of lineages after 50,000 generations is shown for different strengths of directional selection, $G_{y}$, and for different strengths of disruptive selection, $D_{x}=\sigma_{\alpha}^{-2}-\sigma_{K}^{-2}$. The continuous line shows the linear relationship $D_{x}=80 \cdot G_{y}$. Other parameters are as in figure 1, except for $\sigma_{\mu x}=\sigma_{\mu y}=2.5 \times 10^{-3}$.

Figure 3: Evolutionary dynamics in the simplified two-dimensional sexual model. (A) Evolution of reproductive isolation through divergence between male display trait (upper panel) and female preference trait (lower panel). The red curves show the variance between the two subpopulations, while the blue and green curves show the variances within each of these subpopulations. (B) Evolution of the phenotype distribution. The rendering of pixels indicates the corresponding population size (white to black), as well as their average trait value $y$ compared with the community's current average $\bar{y}$ (green to blue to red). At each moment, the subpopulations used in (A) are defined as falling on either side of a line drawn through the mean of the current phenotype distribution $n(x, y)$ and being orthogonal to 
this distribution's direction of maximum variance. Parameters: $K_{0}=500, \sigma_{K}=0.2, \sigma_{\alpha}=0.07, r=1$, $G_{y}=b_{1}=3.5, d_{1}=0, \sigma_{m}=2.83, L_{x}=60, L_{y}=50, L_{m}=L_{p}=20, \mu_{x}=5 \times 10^{-5}, \mu_{y}=10^{-5}$, $\mu_{m}=\mu_{p}=5 \times 10^{-3}, C_{x}=6, C_{y}=5$, and $C_{m}=C_{p}=2$.

Figure 4: Evolutionary dynamics in the full two-dimensional model for (A) asexual reproduction and (B) sexual reproduction. The design of panels (A) and (B) matches that of figures $1 \mathrm{e}$ and $3 \mathrm{~b}$, respectively. Parameters for (A): $K_{0}=100,000, \sigma_{K x}=0.2, \sigma_{K y}=0.5, \sigma_{\alpha x}=0.08, \sigma_{\alpha y}=0.6$, $\sigma_{\mu x}=\sigma_{\mu y}=5 \times 10^{-3}$, and $\mu=2.5 \times 10^{-5}$. The population in (A) is initialized at $y=-1.0$ and reaches a community average of $\bar{y}=-0.62$ at $t=5 \times 10^{6}$. Parameters for $(\mathrm{B}): K_{0}=1,000, \sigma_{K x}=0.2, \sigma_{K y}=0.5$, $L_{x}=60, L_{y}=40, L_{m}=L_{p}=20, \mu_{x}=7 \times 10^{-5}, \mu_{y}=10^{-6}$, and $\mu_{m}=\mu_{p}=5 \times 10^{-3}$; other parameters as in figure 3. The population in (B) is initialized at $y=-1.0$ and reaches a community average of $\bar{y}=-0.28$ at $t=1.2 \times 10^{4}$.

Figure 5: Relationship between the age of lineages and their fitness in trait $y$, for the evolutionary dynamics shown in figure 1e. Ages of lineages are defined by assuming that at a branching of the evolutionary tree (A) both lineages are assigned an age of 0 , or (B) only one lineage, chosen at random, is assigned an age of 0 , while the other lineage inherits its age from the common ancestor. The resultant distributions of lineage lifetimes are depicted at the bottom of each panel, with frequencies as indicated by the right vertical axes. 
(A)

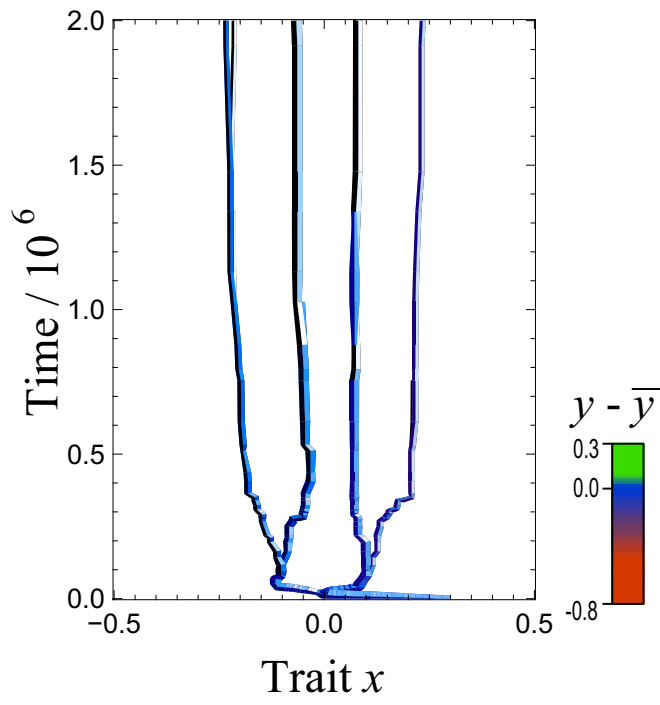

(D)

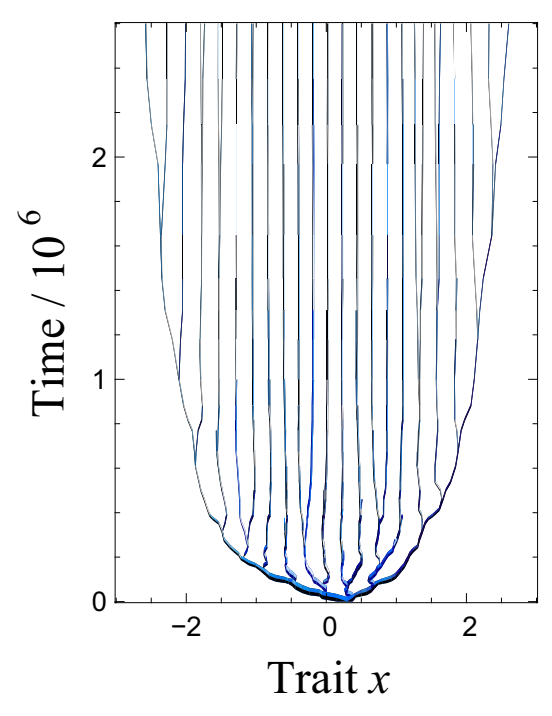

(B)

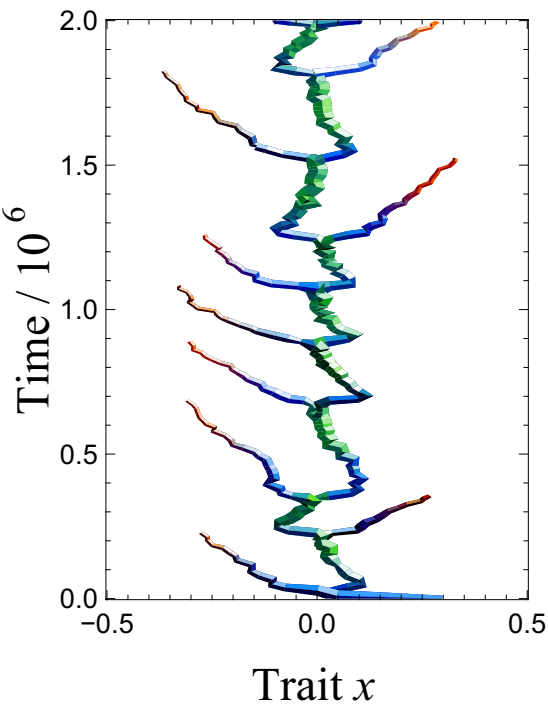

(E)

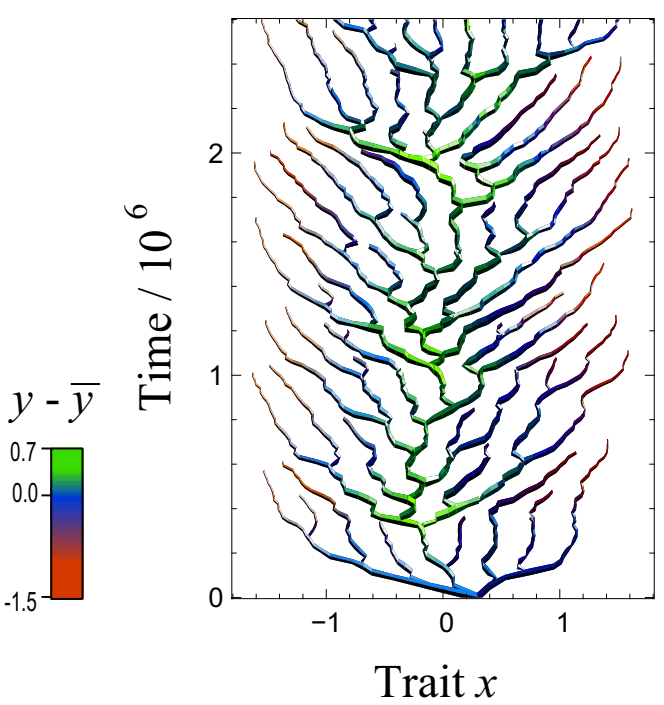

(C)

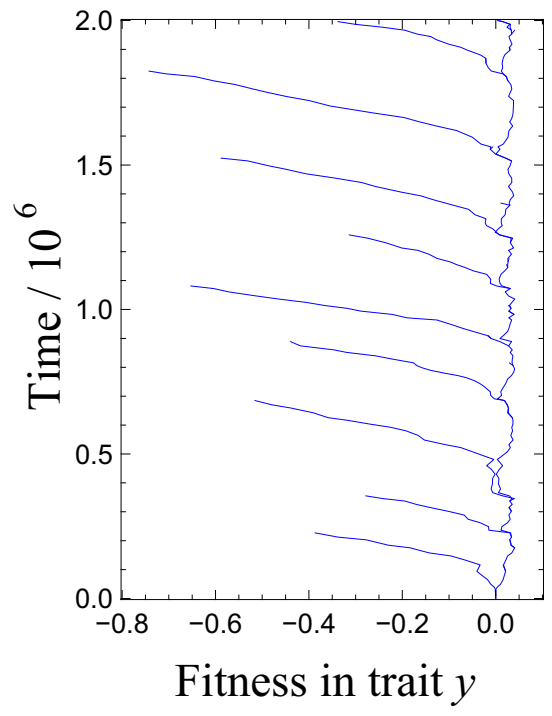

(F)

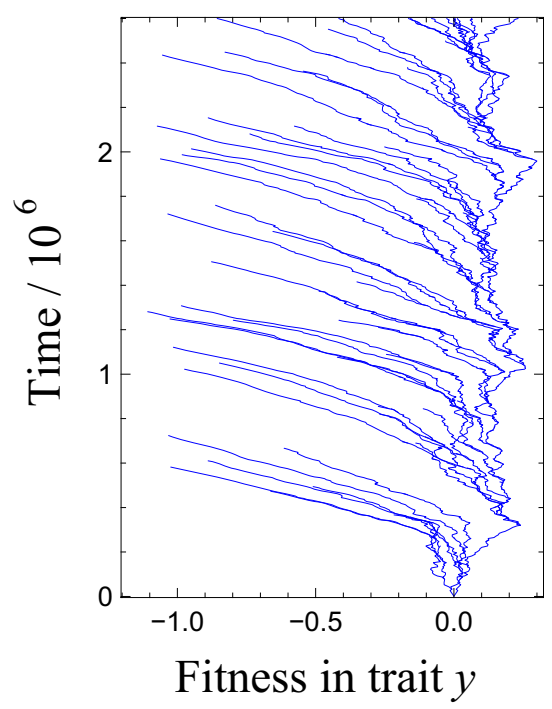




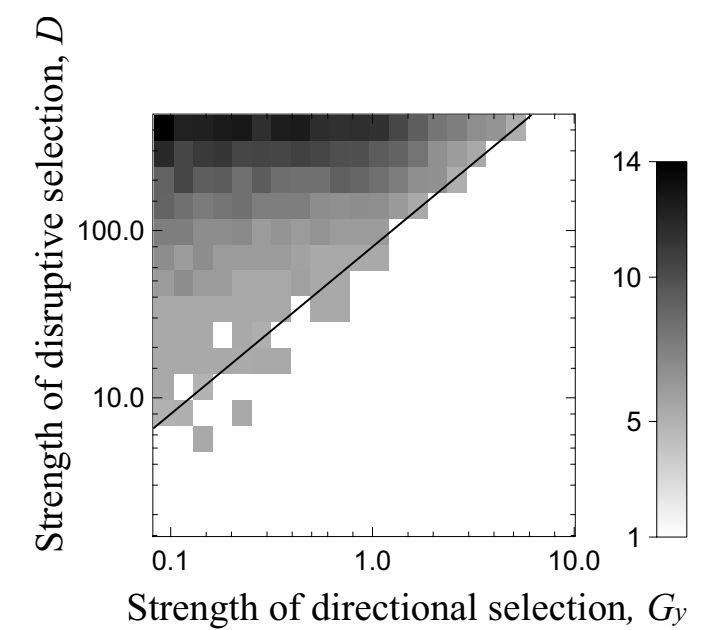


(A)
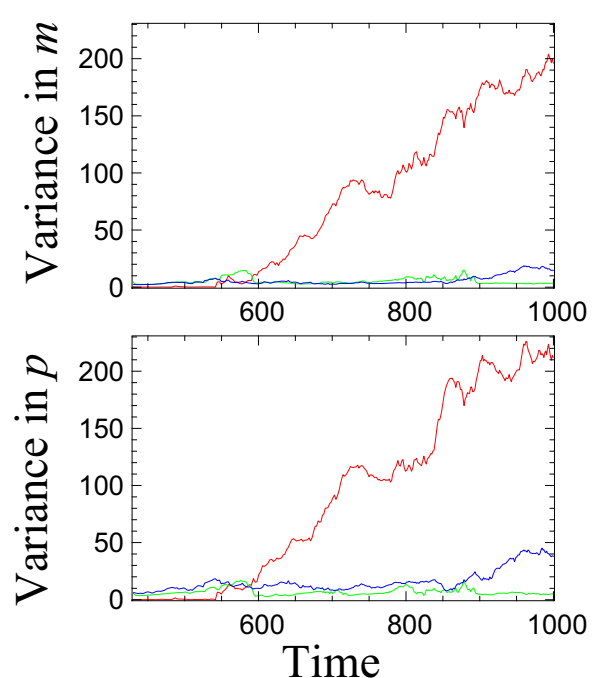

(B)

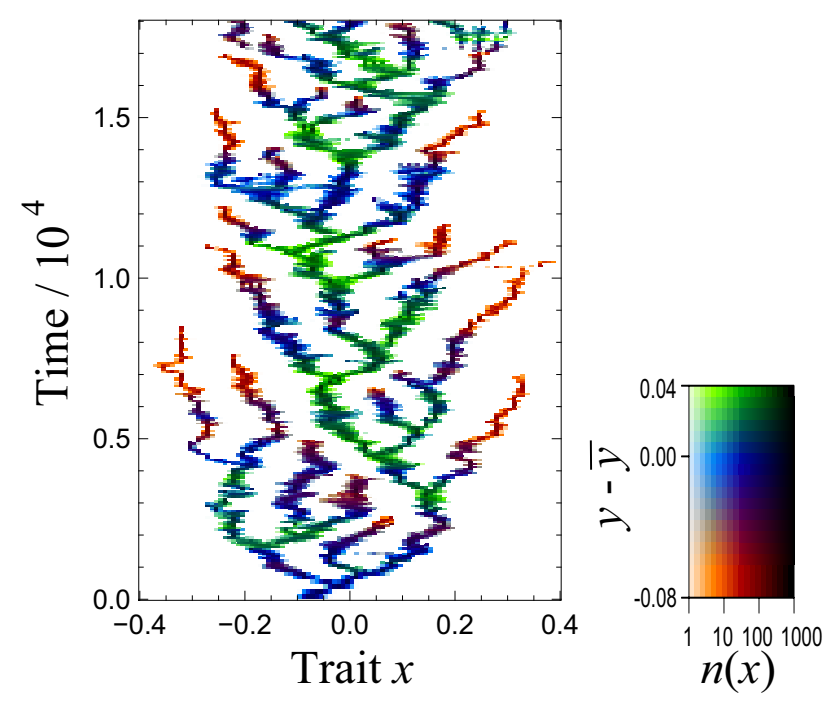



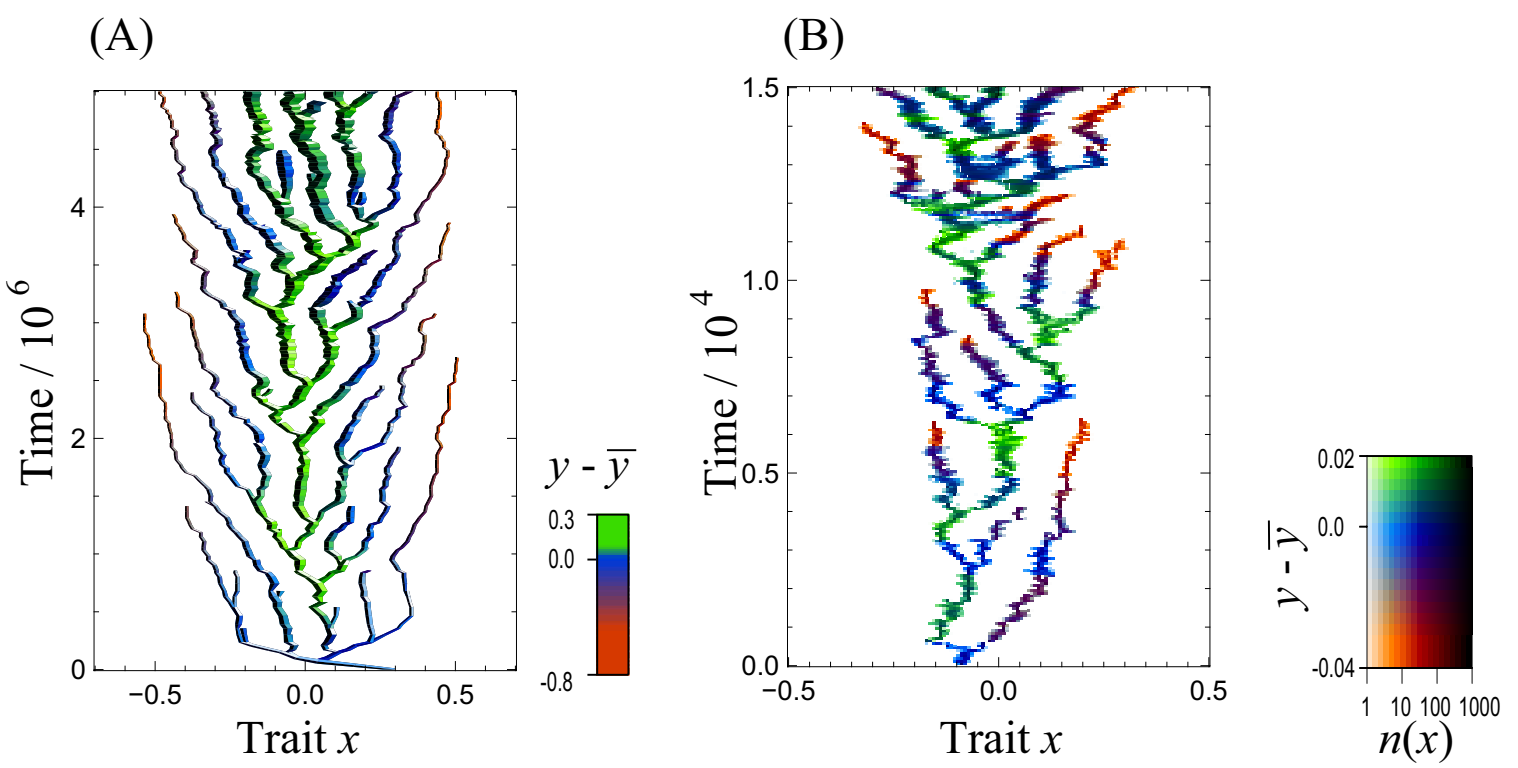
(A)



(B)

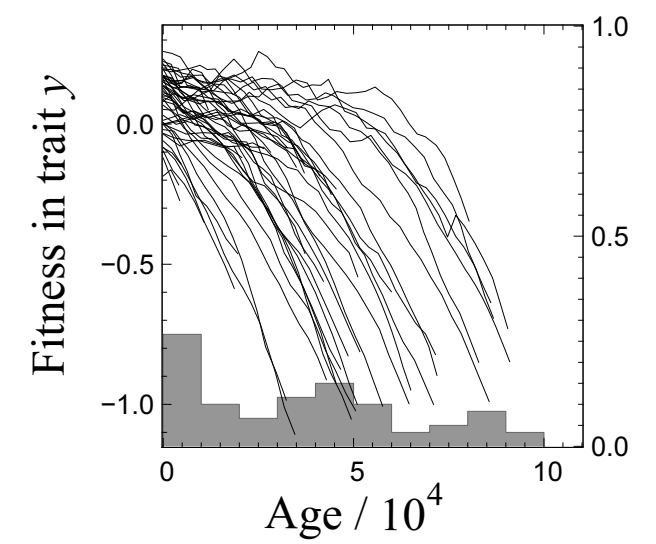

